\title{
Sinopse do gênero Jacquemontia Choisy (Convolvulaceae) no Estado de São Paulo, Brasil: notas nomenclaturais, taxonômicas e geográficas
}

\author{
Mayara Pastore ${ }^{1,2,3,4}$ e Rosângela Simão-Bianchini ${ }^{2}$
}

Recebido: 13.10.2017; aceito: 19.10.2017

\begin{abstract}
Synopsis of the genus Jacquemontia Choisy (Convolvulaceae) in São Paulo State, Brazil: nomenclatural, taxonomic and geographical notes). This taxonomic study is part of the project "Flora Fanerogâmica do Estado de São Paulo". In São Paulo State, Jacquemontia is represented by 14 species and two varieties. These species can be distinguished mainly through their habit, type of trichomes, inflorescences and sepals. Identification key, illustrations, photographs, synonymy, typification, geographical distribution, taxonomic comments, and conservation status are presented for all the studied species. Additionally, eight lectotypes and two neotypes are proposed. Finally, we classified one species as presumably extinct, one as vulnerable, and one as near threatened in São Paulo State.
\end{abstract}

Keywords: Atlantic rain forest, Cerrado, Solanales, typification

RESUMO - (Sinopse do gênero Jacquemontia Choisy (Convolvulaceae) no Estado de São Paulo, Brasil: notas nomenclaturais, taxonômicas e geográficas). O presente estudo taxonômico é parte integrante do projeto "Flora Fanerogâmica do Estado de São Paulo". No Estado de São Paulo, Jacquemontia está representado por 14 espécies e duas variedades. Essas espécies podem ser reconhecidas principalmente por meio do hábito, tipos de tricomas, inflorescências e sépalas. São apresentadas chave de identificação, ilustrações, fotografias, sinonímias, tipificações e distribuição geográfica, além de comentários taxonômicos e estado de conservação de todas as espécies estudadas. Adicionalmente, oito lectótipos e dois neótipos estão sendo aqui propostos. Por fim, uma espécie foi classificada como presumivelmente extinta, uma como vulnerável e uma como quase ameaçada de extinção no Estado de São Paulo.

Palavras-chave: Cerrado, Floresta Atlântica, Solanales, tipificação

\section{Introdução}

Convolvulaceae é considerada cosmopolita com numerosas espécies nos trópicos e poucas nas zonas temperadas (Austin \& Cavalcante 1982, Staples 2012), representada por 60 gêneros e cerca de 1.900 espécies (Cheek \& Simão-Bianchini 2013, Buril et al. 2015, Simões \& Staples 2017, Athiê-Souza et al. 2017).

A família pertence à ordem Solanales, juntamente com Solanaceae, Hydroleaceae, Montiniaceae, e Sphenocleaceae (APG IV 2016). Convolvulaceae e Solanaceae são famílias-irmãs, devido à presença de floema interno, alcaloides quimicamente semelhantes e muitos caracteres moleculares (Stefanović et al. 2002, Olmstead et al. 2008, Judd et al. 2009).
Jacquemontia foi descrito por Choisy (1834), na obra Mémoires de la Société de Physique et d'Histoire Naturelle de Genève, em homenagem ao naturalista francês Victor Jacquemont. Nenhuma espécie foi descrita para o gênero nessa obra, Choisy (l.c.) apenas ilustrou uma espécie de Jacquemontia e indicou que as espécies de Convolvulus L. com estigmas elipsoides deveriam ser transferidas para Jacquemontia, exemplificando com C. azureus Rich. e C. coeruleus Schum. \& Thonn. O primeiro tratamento formal para o gênero foi realizado por Choisy (1838), incluindo 11 espécies e seis variedades.

O gênero apresenta cerca de 120 espécies, a maioria ocorre na zona Neotropical, e poucas espécies na África, Ásia e Oceania (Rhui-Cheng \& Staples

1. Parte da Dissertação de Mestrado da primeira Autora

2. Instituto de Botânica, Núcleo de Pesquisa Curadoria do Herbário, Avenida Miguel Stéfano, 3687, Água Funda, 0431-192 São Paulo, SP, Brasil

3. Instituto Tecnológico Vale, Rua Boaventura da Silva, 955, Nazaré, 66055-090 Belém, PA, Brasil

4. Autor para correspondência: pastoremay@gmail.com 
1995, Staples et al. 2008, Staples 2012). No Brasil o gênero está representado por 67 espécies, entre as quais 43 são consideradas endêmicas, encontradas em todos os biomas brasileiros (Flora do Brasil 2020, em constr.).

Desde o trabalho de Choisy (1834), Jacquemontia foi tradicionalmente mantido na tribo Convolvuleae. Os estudos filogenéticos de Stefanović et al. (2002) baseados em dados moleculares e na classificação da família proposta por Austin $(1973,1998)$, apontaram Convolvuleae como polifilética, incluindo os gêneros Jacquemontia, Calystegia R.Br., Convolvulus e Polymeria R.Br. Logo após, Stefanović et al. (2003) propuseram uma nova classificação filogenética para a família, estabelecendo o monofiletismo de Convolvuleae ao excluir Jacquemontia dessa tribo e ao inserir o gênero na nova tribo Jacquemontieae.

Stefanović et al. (2002) concluíram que o gênero é monofilético e muito bem suportado por caracteres moleculares. Os estudos filogenéticos de Jacquemontia realizados por Buril (2013), que amostrou cerca de $50 \%$ das espécies, também confirmam o monofiletismo do gênero, desde que algumas espécies sejam excluídas. Buril et al. (2015) transferiram Jacquemontia montana Moric., para o novo gênero Daustinia Buril \& A.R. Simões.

Jacquemontia é representado por trepadeiras volúveis, menos frequentemente ervas ou subarbustos. Os tricomas são estrelados 3-8-radiados ou derivados destes, raramente simples ou glandulares. As sépalas são distintas em tamanho e forma ou todas semelhantes entre si, e persistentes no fruto. O gineceu possui ovário glabro, estilete simples com dois estigmas elipsoides a oblongos e achatados dorsiventralmente. As cápsulas são deiscentes, geralmente com oito valvas, as sementes são glabras, verrucosas a estriadas, e em geral com margens curtamente aladas (O'Donell 1960a, Robertson 1971, Simão-Bianchini 2009).

Várias espécies de Jacquemontia possuem muitas inflorescências por indivíduo e longos pedúnculos (até $24 \mathrm{~cm}$ compr.) que proporcionam a exteriorização das flores. A associação desses caracteres aumenta a atratividade para o polinizador, através da grande quantidade de flores disponíveis (Piedade-Kiill \& Ranga 2000). Muitas espécies também apresentam corola com porção basal e áreas mesopétalas mais claras que segundo Piedade-Kiill \& Ranga (2000) funcionam como guias de néctar.

O presente estudo teve como objetivo realizar estudos taxonômicos das espécies de Jacquemontia no Estado de São Paulo, contribuindo para a conclusão da monografia de Convolvulaceae da "Flora Fanerogâmica do Estado de São Paulo". Além de enriquecer e atualizar as coleções de Convolvulaceae nos principais herbários do Estado de São Paulo, fornecendo também informações para a conservação da flora.

\section{Material e métodos}

O Estado de São Paulo localiza-se na região Sudeste do Brasil. Formado por uma vegetação diversificada, que inclui a Mata Atlântica representada pela Floresta Ombrófila Densa na Serra do Mar, se estendendo para o planalto em variadas formas de Florestas Mesófilas Semidecíduas. As áreas abertas da região central e do oeste são dominadas pelos Cerrados, incluindo desde os Campos Sujos até Cerradões. Em áreas menores ocorrem Restingas, Dunas, Manguezais, além das Florestas AltoMontanas e Campos de Altitude acima de $2.000 \mathrm{~m}$ na Serra da Mantiqueira (Wanderley et al. 2005).

A sinopse taxonômica de Jacquemontia aqui apresentada é parte do projeto "Flora Fanerogâmica do Estado de São Paulo". Este trabalho foi baseado em coleções dos principais herbários do Estado de São Paulo: BOTU, ESA, HRCB, IAC, PMSP, SJRP, SP, SPF, SPSF, UEC, e coleções importantes de outros grandes herbários: HB, K, MBM, P, R e RB (Thiers continuamente atualizado). Além das imagens digitais disponibilizadas por diversos outros herbários e observação das espécies em campo em diferentes regiões do Estado.

Uma chave de identificação para as espécies de Jacquemontia no Estado de São Paulo é apresentada e para cada espécie são referidos obra príncipes, materiais-tipo, sinonímias, comentários taxonômicos, distribuição geográfica, estado de conservação e materiais selecionados. Os mapas com pontos de ocorrência das espécies no Estado de São Paulo foram elaborados através do programa QGIS 2.18 (2016), pelo geógrafo Rafael Melo de Brito. As avaliações de conservação a nível estadual foram realizadas através da metodologia elaborada por Mamede et al. (2007), adaptada a partir das categorias propostas pela IUCN (2001). Para apresentação dos materiais examinados foram selecionados um por município.

Fotografias em campo e ilustrações com base em materiais herborizados e conservados a álcool são incluídas para algumas espécies. Os desenhos a grafite foram realizados pela primeira autora com utilização de estereomicroscópio com câmara-clara acoplada, e 
cobertos a nanquim sobre papel vegetal pelo biólogo Vitor Gedha Pescara.

\section{Resultados e Discussão}

O Estado de São Paulo está representado por 14 espécies e duas variedades de Jacquemontia: $J$. blanchetii Moric. var. blanchetii, J. blanchetii Moric. var. major Choisy, J. cataractae Krapov., J. gabrielii (Choisy) Buril, $J$. densiflora (Meisn.) Hallier f., $J$. ferruginea Choisy, J. frankeana (Schltdl.) M. Pastore \& Sim.-Bianch., J. glabrescens (Meisn.) M. Pastore \& Sim.-Bianch., J. holosericea (Weinm.) O'Donell, J. selloi (Meisn.) Hallier f., J. spiciflora (Choisy) Hallier f., J. sphaerocephala Meisn., J. sphaerostigma (Cav.) Rusby, J. tamnifolia (L.) Griseb. e J. velutina Choisy.

$\mathrm{O}$ presente estudo proporcionou uma melhor delimitação taxonômica de Jacquemontia no Estado de São Paulo, já que anteriormente foram realizados apenas levantamentos e um breve tratamento taxonômicos para Convolvulaceae no Estado.

O primeiro tratamento foi realizado por Falcão (1971), considerado uma compilação de dados com descrições bem sucintas para as espécies de Convolvulaceae, incluindo nove espécies de Jacquemontia. No mesmo trabalho, J. evolvuloides (Moric.) Meisn. e $J$. martii foram reconhecidas para o Estado de São Paulo, mas a primeira foi baseada no material H. Leitão-Filho 664 (IAC, RB, SP) que trata-se de $J$. ferruginea, e a segunda baseada nos espécimes M. Kuhlmann 306 (SP) e A.P. Viegas \& C.P. Viegas 3797 (SP) que correspondem à J. blanchetii.

Um segundo levantamento de Convolvulaceae foi realizado por Simão-Bianchini \& Silva (2011) para o checklist de Spermatophyta do Estado de São Paulo, no qual reconheceram 18 espécies, entre estas oito foram revistas.

Jacquemontia acrocephala Meisn., conhecida apenas pelo tipo coletado em São Paulo, no presente estudo está sendo considerada como sinônimo de $J$. sphaerocephala.

Anteriormente alguns espécimes (ex. Barbiellini s.n. [RB 30000]) estavam determinados como Jacquemontia glaucescens Choisy, mas são aqui reconhecidos como $J$. holosericea ou $J$. frankeana. Essas três espécies são morfologicamente similares, sendo que $J$. glaucescens é conhecida apenas na Bahia, Espírito Santo e Rio de Janeiro.

Jacquemontia grandiflora Meisn. está sendo considerada como sinônimo de $J$. ferruginea, assim como reconheceu Hallier (1899). Enquanto, os espécimes anteriormente determinados como $J$. grandiflora var. glabrescens Meisn. (ex. Hashimoto 17392 [SP]), estão sendo reconhecidos como $J$. glabrescens, assim como Pastore \& Simão-Bianchini (2015) propuseram a mudança de status.

Espécimes anteriormente determinados como $J$. martii Choisy (ex. Sampaio 4326 [R]) eram assim reconhecidos devido às sépalas com ápice agudo, mas aqui estão sendo determinados como $J$. blanchetii Moric. var. major Choisy.

Algumas exsicatas de J. densiflora (Meisn.) Hallier f. (ex. Bianchini 3 [SPF]) estavam determinadas como J. polyantha (Schltdl. \& Cham.) Hallier f., espécie encontrada no México.

O espécime Luederwaldt \& Fonseca 10910 (SP), coletado na Ilha de Alcatrazes em Santos, estava identificado como J. rufa (Choisy) Hallier f., porém trata-se de um representante de $J$. ferruginea.

Jacquemontia lasioclados (Choisy) O'Donell foi registrada para São Paulo, baseada no espécime Glaziou 11.267 (R) referido para os campos da Bocaina. No entanto, seria a única amostra da espécie para o Estado, pois todas as demais se restringem ao norte de Minas Gerais em área de campos rupestres, onde a espécie é reconhecida como endêmica.

Jacquemontia hallieriana Ooststr ( $\equiv J$. cephalantha Hallier f.) também foi referida para São Paulo na Serra da Bocaina, a partir do síntipo Glaziou 19.670 (B, K). Entretanto, existe outro espécime coletado por Glaziou com o mesmo número, depositado no herbário R, referido para a vila de Biribiri, Diamantina em Minas Gerais. A distribuição de $J$. cephalantha Hallier f. é conhecida apenas para o norte de Minas Gerais e sul da Bahia.

Um terceiro espécime coletado por Glaziou (s.n., P03545420), também referido para Serra da Bocaina em São Paulo, trata-se de Jacquemontia heterotricha O’Donell, espécie encontrada em áreas campestres do Norte de Minas Gerais e Goiás, com disjunção no Rio Grande do Sul e Argentina, mas não se conhece nenhuma outra coleta para São Paulo.

Várias expedições foram realizadas para a Serra da Bocaina, mas nenhuma das três espécies supracitadas foram encontradas. Wurdack (1970) identificou uma problemática com as coletas de Melastomataceae realizadas por Glaziou, uma vez que vários espécimes possuem números trocados e localidades equivocadas. Considerando que também houve erro na descrição de localidade das coletas de Glaziou, J. lasioclados, $J$. hallieriana e $J$. heterotricha não foram aqui incluídas como nativas do Estado de São Paulo. 
Chave para as espécies de Jacquemontia no

Estado de São Paulo

1. Subarbustos eretos ou com ramos apicais escandentes; folhas com base arredondada, cuneada ou atenuada

2. Inflorescências espiciformes; sépalas de tamanho igual a subigual, as internas ligeiramente maiores que as externas, ápice arredondado a obtuso, as externas glabras ou com tricomas restritos ao ápice e região central, ciliadas ou margens glabras

12. J. spiciflora

2. Inflorescências corimbiformes ou glomeruliformes; sépalas de tamanho desigual, as internas menores que as externas, ápice acuminado, as externas tomentosas ou velutinas

3. Inflorescências glomeruliformes, multifloras (acima de 30 flores), pedúnculos 2-4 mm compr.; sépalas externas 13-16 mm compr., rômbicas; grãos de pólen tricolpados ... 10. J. sphaerocephala

3. Inflorescências corimbiformes, 3-5-floras, pedúnculos 2,7-6 cm compr.; sépalas externas 9-10 mm compr., ovadas; grãos de pólen pantocolpados 9. J. selloi

1. Trepadeiras volúveis (J. tamnifolia pode ser ereta quando jovem); folhas com base cordada, subcordada, truncada ou obtusa

4. Sépalas hirsutas; indumento dos ramos formado por tricomas estrelados 3-radiados com o raio central mais longo, forcados ou tricomas simples, entremeados ou não a tricomas forcados ou estrelados 3-radiados com raios iguais a subiguais

5. Dicásios umbeliformes a corimbiformes, bractéolas subiguais entre si; tricomas glandulares presentes 11. J. sphaerostigma

5. Dicásios glomeruliformes, bractéolas desiguais em cada verticilo, as inferiores foliáceas; tricomas glandulares ausentes 13. J. tamnifolia

4. Sépalas pubescentes ou glabras; indumento dos ramos formado apenas por tricomas estrelados 3-radiados ou 4-5-radiados com raios iguais a subiguais

6. Sépalas externas mais longas que as internas, ápice agudo a acuminado

7. Corola 0,6-0,7 cm compr., rotáceo-infundibuliforme; bractéolas subiguais entre si

7. Corola 1,2-3,3 cm compr., infundibuliforme; duas bractéolas inferiores foliáceas, maiores que as superiores

8. Sépalas externas lanceoladas; pedicelos glabrescentes a glabros

2.J. cataractae

8. Sépalas externas rômbicas a ovadas; pedicelos tomentosos a velutinos

9. Folhas com ápice acuminado; sépalas glabras a glabrescentes, as externas 9-13×4,5-6 mm, as internas 7-9 $\times 5-5,5 \mathrm{~mm}$ 7.J. glabrescens

9. Folhas com ápice obtuso a agudo; sépalas tomentosas a vilosas, as externas 7-11 ×2,5-5 mm, as internas $5-7 \times 2-3 \mathrm{~mm}$ 4. J. ferruginea

6. Sépalas externas mais curtas que as internas ou sépalas iguais entre si, ápice truncado, arredondado ou obtuso (ápice agudo em $J$. blanchetii var. major)

10. Plantas com tricomas estrelados 4-5-radiados; sépalas externas menores que as internas

11. Face abaxial das folhas pubérula a glabrescente, ambas as faces com tricomas de raios adpressos 5. J. frankeana

11. Face abaxial das folhas tomentosa a pubescente, face adaxial com tricomas de raios eretos ou adpressos, abaxial com tricomas de raios eretos ... 8. J. holosericea

10. Plantas com tricomas estrelados 3-radiados; sépalas de tamanho igual a subigual

12. Cálice com sépalas pubescentes; áreas mesopétalas glabras

14. J. velutina

12. Cálice com sépalas glabras ou apenas ciliadas; áreas mesopétalas com tricomas simples apenas no ápice

13. Ramos e folhas hirsutos; face adaxial da folha com tricomas de raios desiguais, $o$ central longo (1-1,5 mm compr.) e dois laterais curtos (ca. 0,2 mm compr.); dicásios corimbiformes; grãos de pólen pantocolpados ... 6. J. gabrielii

13. Ramos e folhas pubescentes a glabros; face adaxial da folha com tricomas de raios iguais a subiguais $(0,2-0,4 \mathrm{~mm}$ compr.); dicásios umbeliformes; grãos de pólen tricolpados 
1. Jacquemontia blanchetii Moric., P1. Nouv. Amér. 27: 41. 1838. $\equiv$ Montejacquia blanchetii (Moric.) Roberty, Candollea 14: 33. 1952. Tipos: BRASIL. BAHIA: J.S. Blanchet 1165 (lectosíntipo foto G!, designado por Buril \& Staples [2017]); J.S. Blanchet 104 (lectosíntipo foto G!); J.S. Blanchet 564 (lectosíntipo foto G!). Convolvulus blanchetii Steud., Nomencl. Bot. (ed. 3) 2(1): 407. 1840, nom. nud.

Figura 2

Espécie caracterizada pelos ramos e folhas esparsamente pubescentes a glabros inflorescências umbeliformes, sépalas ovadas a obovadas, glabras, semelhantes em tamanho e forma, ou as externas ligeiramente menores que as internas.

Muito semelhante morfologicamente à $J$. martii, especialmente por ambas possuírem inflorescência umbeliforme e sépalas glabras. No entanto, $J$. blanchetii possui sépalas com ápice arredondado a obtuso, enquanto $J$. martii possui sépalas com ápice acuminado e revoluto (figura $1 \mathrm{a}-\mathrm{b}$ ), e sua distribuição geográfica se restringe a Alagoas, Ceará, Pernambuco, Bahia, Minas Gerais e Espírito Santo.

De acordo com a metodologia de Mamede et al. (2007), esta espécie foi classificada como menos preocupante (LC) no Estado de São Paulo, pois não se enquadra em nenhum dos 11 critérios utilizados.

Duas variedades estão sendo aqui consideradas, Jacquemontia blanchetii var. blanchetii e J. blanchetti var. major Choisy. Enquanto J. blanchetii var. puberula Hassl. consideramos como sinônimo da variedade típica, por ser diferenciada apenas pelo indumento pubérulo. Em 1953, O’Donell já havia reconhecido $J$. blanchetii var. puberula como sinônimo de J. blanchetii, ao determinar o isótipo depositado no herbário BM.

Chave para as variedades de Jacquemontia blanchetii

1. Sépalas ovadas a obovadas com ápice arredondado a obtuso ... 1.1.J. blanchetii var. blanchetii

1. Sépalas obovadas a oblongas com ápice agudo ..... 1.2.J. blanchetii var. major

\subsection{Jacquemontia blanchetii var. blanchetii.} Ipomoea filipedunculata Rusby, Bull. Torrey Bot. Club. 150. 1899. Tipo: BOLÍVIA. Guanai, V-1886, H.H. Rusby 1997 (holótipo NY!). Jacquemontia blanchetii Moric. var. puberula Hassl., Repert. Spec. Nov. Regni Veg. 9: 160. 1911. Tipo: PARAGUAI. Presidente Hayes: Gran Chaco, Santa Elisa, 1905, E. Hassler 2752 (holótipo G; isótipo foto BM!).

Figuras $1 \mathrm{c}, 3$ a-c, 5 a-f
Encontrada no norte da Argentina, Paraguai, Bolívia, Peru, e Brasil nos Estados de Rondônia, Mato Grosso, Mato Grosso do Sul, Goiás, Minas Gerais, Bahia, Rio de Janeiro, São Paulo, Paraná e Santa Catarina (O'Donell 1960b; Flora do Brasil 2020, em constr.). Em São Paulo ocorre no Cerrado e Floresta Estacional Semidecídua, em bordas de mata. Coletada com flores e frutos de novembro a agosto.

Material examinado selecionado: BRASIL. São Paulo: Amparo, Três Pontes, 28-V-1927, F.C. Hoehne 20585 (SP); Angatuba, Estrada para Itatinga, ca. $16 \mathrm{~km}$ de Angatuba, 2325'10"S, 48³0'16"W, 27-1-1996, V.C. Souza et al. 10696 (SP, ESA); Botucatu, Morro do Rubião, 22 ${ }^{\circ} 31^{\prime} 58^{\prime \prime} \mathrm{S}, 48^{\circ} 1741 \mathrm{~W}, 7-3-2013$, M. Pastore S.A. Adachi 271 (SP, BOTU, IAC); Buri, Estação Experimental do Instituto Florestal, 8-XII-1987, O.C. Goes \& N. Figueiredo 427 (RB); Campinas, 1896, J.C. Novaes 3751 (SP); Eldorado, fragmento de mata, próximo ao portal da cidade, 9-XII-2012, E.P. Fortes 1 (SP); Ipeúna, Ribeirão Passa-Cinco, 26-I-1984, N.H.Fraldi 166 (UEC); Itapetininga, Estação Experimental de Itapetininga, 2323'32"S, 48 00'28"W, 18-XII-2013, M. Pastore et al. 208 (SP, ESA, HRCB, IAC, PMSP, SJRP SPSF); Itapira, 12-I-1994, K.D. Barreto et al. 1791 (SP, ESA); Itu, Area de Proteção Ambiental de Itu, 8-I-1987, P.B. Cuezzo 25193 (R); Jundiaí, Fazenda Malota Fraldas da Serra do Japi, 9-V-1977, R.P. Belém 38 (UB); Lindóia, 21-I-1939, A.P. Viegas C.P. Viegas 3797 (SP); Magda, Fazenda CFM (ingleses), 17-V-1995, L.C. Bernacci et al. 1772 (SP, SPF, UEC, HRCB); Monte Alegre do Sul, 24-III-1943, M. Kuhlmann 306 (SP); Morungaba, Observatório Capricórnio, 9-I-1986, N. Taroda K. Yamamoto 18302 (UEC, MBM); Paranapanema, 5-XII-2007, J.B. Baitello et al. 1940 (SP, SPSF); Paulo Faria, Estação Ecológica de Paulo de Faria, 22-III-1994, O.S. Ribas 280 (MBM); Pereira Barreto, divisa com Mirandópolis, Fazenda Esmeralda, 20³8'18"S, 51 ${ }^{\circ} 06^{\prime} 33^{\prime \prime} \mathrm{W}, 2$-VIII-1995, M.R. Pereira-Noronha et al. 1162 (SP); Potirendaba, Liana, 30-3-1999, R.G.V. Camacho C.R. Tonetti; J.C. Souza 1 (SPF); Rio Claro, Campos da UNESP Bela Vista, 29-V-2007, R. Lima 172 (HUEFS, JPB); São Carlos, 2-VIII-1888, A. Loefgren 815 (SP); São José do Rio Preto, 12-IX-1996, R.P. Lyra-Lemos 528 (HUEFS, MAC); São Roque, SP-280, 3-III-1992, G. Hashimoto 20496 (SP); Socorro, 7-III-2000, M. Groppo Jr. 368 (SP, SPF); Sorocaba, Votorantin, 17-IV-1995, R. Simão-Bianchini et al. 652 (SP, SPF, ESA, UEC, HRCB); Tietê, próximo à estação, 
27-IV-1995, L.C. Bernacci et al. 1592 (SP, UEC, IAC, PMSP).

Ilustrações: Moricand (1838, t. 27); O'Donell (1960a, p. 7, fig. 1 A-J); Ferreira (2013, p. 293, fig.1 A-D).

1.2. Jacquemontia blanchetii var. major Choisy, in DC. Prodr. Syst Nat. 9: 398. 1845. EJacquemontia martii Choisyvar.floribunda Meisn., in Mart.,Fl.Bras. 7: 298. 1869. Tipo: BRASIL. "Rio de St. Francisco", C.F.P. Martius s.n. (lectótipo foto M[184714]!, aqui designado; isolectótipos foto M[84711]!, foto M[84712]!, foto M[184713]!) syn. nov.

Figura $1 \mathrm{~b}$

No herbário M há quatro exemplares do tipo de $J$. blanchetii var. major, todos possuem a determinação de Choisy. Selecionamos como lectótipo o exemplar M 184714, cuja localidade está referida como em seu protólogo e o mais semelhante com a ilustração de Meisner (1869).

Choisy (1845) reconheceu J. blanchetii var. major, baseada no material Martius s.n., coletado na região do Rio São Francisco (sem referência de localidade específica), e a diferenciou da variedade típica pelas sépalas com ápice obtuso (Fig. 1 b) e flores ligeiramente maiores. Meisner (1869) examinou o mesmo material e o reconheceu como J. martii var. floribunda Meisn. No presente estudo consideramos a proposta inicial de Choisy e consideramos J. martii var. floribunda como sinônimo de J. blanchetii var. major.

Encontrada apenas no Brasil, bastante comum nos Estados do Espírito Santo e Rio de Janeiro em bordas de Floresta Ombrófila e Floresta Estacional Semidecídua, sendo menos frequente em São Paulo. Coletada com flores em abril.
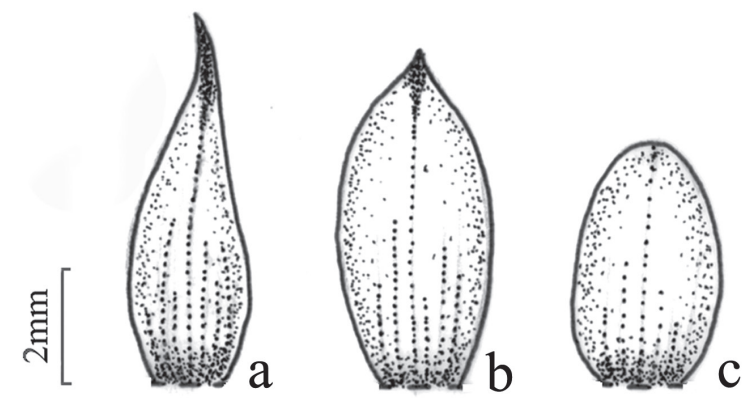

Figura 1. Morfologia das sépalas. a. Jacquemontia martii. b. Jacquemontia blanchetii var. major. c. Jacquemontia blanchetii var. blanchetii.

Figure 1. Morphology of sepals. a. Jacquemontia martii. b. Jacquemontia blanchetii var. major. c. Jacquemontia blanchetii var. blanchetii.
Material examinado selecionado: BRASIL. SÃo Paulo: Horto Florestal de Loreto, IV-1926, A.J. Sampaio 4326 (R).

Material examinado adicional: BRASIL. EsPíRITo Santo: Nova Venécia, V-2008, A.M.A. Amorim et al. 7424 (SP, RB). Rio De Janeiro: Rio de Janeiro, VII-2011, M.G. Bovini \& M. Faria 3563 (RB); Rio de Janeiro, XII-2004, M.D.M. Vianna Filho 425 (RB); Itaipuaçú, IV-1982, R.H.P. Andreata 411 (RB); Niterói, IV-2011, M.C.F. dos Santos 517 (RB).

2. Jacquemontia cataractae Krapov., Bonplandia 18(1): 57-64. 2009. Tipo: ARGENTINA. MisIonEs: Dep. Iguazú, Parque Nacional Iguazú, Cataratas del Iguazú, 10-I-1970, A. Krapovickas \& C.L. Cristóbal 15608 (holótipo foto CTES!).

Figuras 2, $3 \mathrm{~d}-\mathrm{f}$

Espécie frequentemente determinada como Jacquemontia martii, entretanto os ramos e pedúnculos mais espessos, folhas pubescentes, bractéolas foliáceas com 9-20 mm compr. e pedicelos 2-4 mm compr. caracterizam $J$. cataractae. Enquanto J. martii apresenta ramos e pedúnculos delgados, folhas glabras a glabrescentes, bractéolas não foliáceas com até $3 \mathrm{~mm}$ compr. e pedicelos de 4-8 mm compr.

Também pode ser confundida com J. glabrescens, que se diferencia pelos pedicelos tomentosos a velutinos e pelas sépalas externas rômbicas a ovadas, maiores com 7-9 × 5-5,5 mm. Enquanto J. cataractae apresenta pedicelos glabrescentes a glabros e sépalas externas lanceoladas com 7-7,5 × 2,5-3 mm.

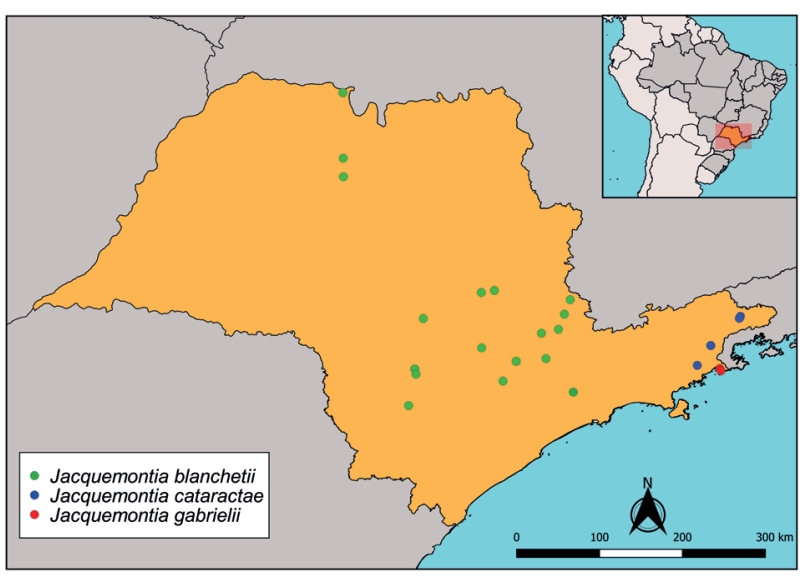

Figura 2. Mapa com a distribuição de Jacquemontia blanchetii, Jacquemontia cataractae e Jacquemontia gabrielii no Estado de São Paulo, Brasil.

Figure 2. Distribution map of Jacquemontia blanchetii, Jacquemontia cataractae and Jacquemontia gabrielii in São Paulo State, Brazil. 

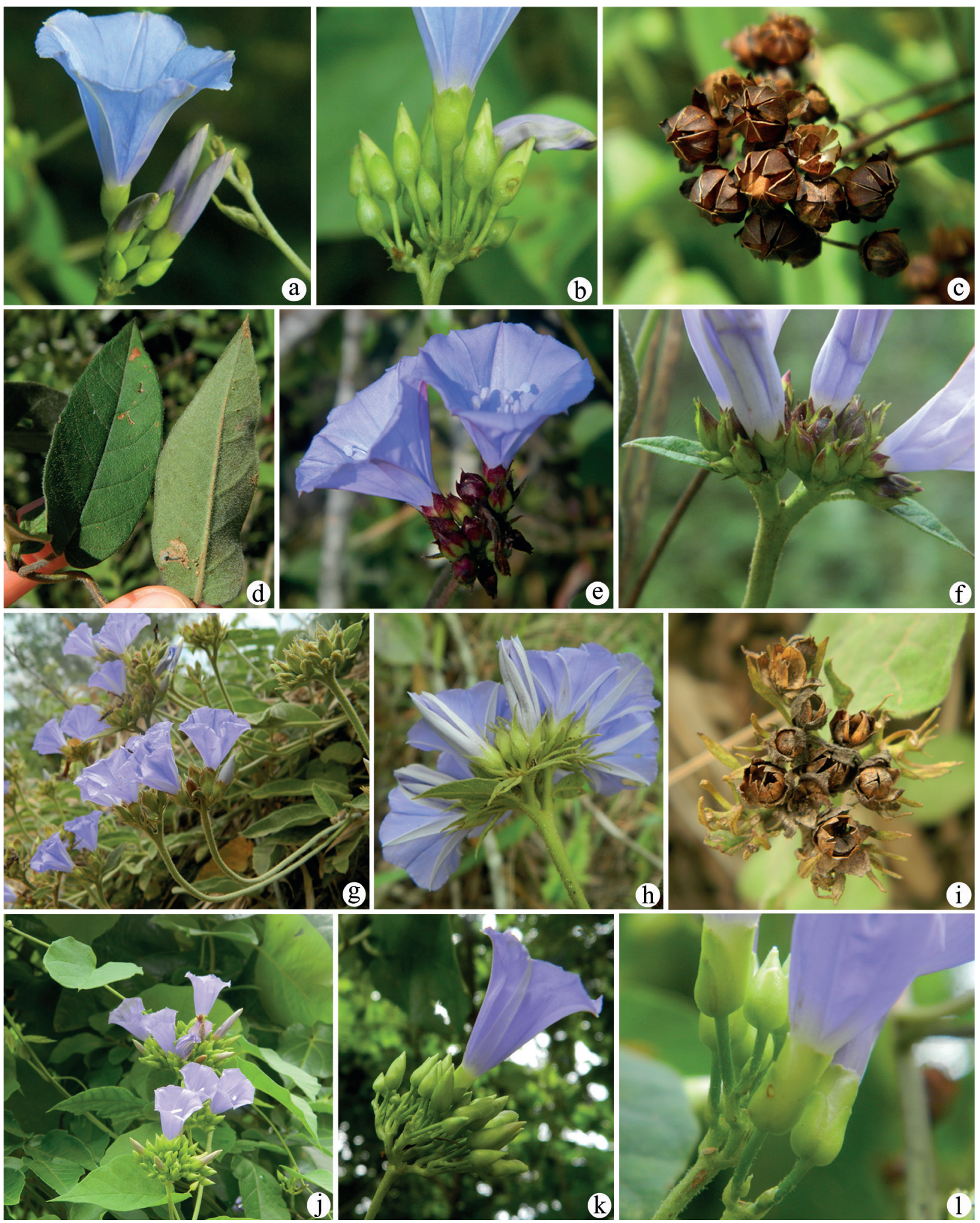

i

Figura 3. a-c. Jacquemontia blanchetii. a. Inflorescência. b. Detalhe dos cálices. c. Cápsulas. d-f. Jacquemontia cataractae. d. Folhas. e. Inflorescência. f. Detalhe dos cálices. g-i. Jacquemontia ferruginea. g. Hábito. h. Inflorescência. i. Cápsulas. j-1. Jacquemontia frankeana . j. Hábito. k. Inflorescência. 1. Detalhe dos cálices.

Figure 3. a-c. Jacquemontia blanchetii. a. Inflorescence. b. Detail of the calyxes. c. Capsules. d-f. Jacquemontia cataractae. d. Leaves. e. Inflorescence. f. Detail of the calyxes. g-i. Jacquemontia ferruginea. g. Habit. h. Inflorescence. i. Capsules. j-1. Jacquemontia frankeana . j. Habit. k. Inflorescence. 1. Detail of the calyxes. 
Outra espécie morfologicamente próxima é Jacquemontia acuminata Rusby, com distribuição restrita na Bolívia, que se diferencia de $J$. cataractae pelas folhas maiores com 8-12 × 4-6 cm $(v s$. 3-7,5 × 1,2-3,1 cm), ausência de bractéolas foliáceas, pedicelos pubescentes a hirsutos, e sépalas mais estreitas de 1,5-2 mm larg.

Jacquemontia cataractae era conhecida apenas para o Parque Nacional Iguazú na Argentina e nas proximidades das Cataratas do Iguaçu no Paraguai (Krapovickas 2009). Recentemente foi registrada para o Brasil (Pastore \& Simão-Bianchini 2015), ocorrendo nos Estados de Minas Gerais, São Paulo e Paraná. Em São Paulo foi encontrada na Serra da Bocaina e proximidades, crescendo nas bordas da Floresta Ombrófila. Coletada com flores e frutos de outubro a abril.

Classificada como menos preocupante (LC) no Estado de São Paulo de acordo com a metodologia de Mamede et al. (2007), pois enquadra-se apenas no critério 9, ou seja, com distribuição restrita a um tipo de vegetação.

Material examinado selecionado: BRASIL. São Paulo: Cunha, XI-1994, G. Hashimoto 20506 (SP). São José do Barreiro, I-2013, M. Pastore et al. 226 (SP, SPSF). São Luiz do Paraitinga, XI-2001, Disciplina Princípios em Taxonomia Vegetal 165 (SPSF, HRCB).

Material examinado adicional: BRASIL. PARANÁ: Campo Largo, Bateias, II-2011, P.P.A. Ferreira et al. 617 (ICN, SP).

Ilustrações: Krapovickas (2009, p. 60, fig. 2 A-J).

3. Jacquemontia densiflora (Meisn.) Hallier f., Bot. Jahrb. Syst. 16: 543.1893 EJacquemontia violacea (Vahl) Choisy var. densiflora Meisn., in Mart., Fl. Bras. 7: 296. 1869. Tipo: BRASIL. Minas Gerais: Lagoa Santa, J..E.B. Warming s.n. (lectótipo foto $\mathrm{BR}[0000005792948]$ ! aqui designado; isolectótipos foto BR[0000005792610]!, foto C!, NY!, P!); GoiÁs: Rio Paranatinga, J.B.E. Pohl 1761 (lectosíntipos B destruído, fragmento foto F!, P!, W).

Jacquemontia rusbyana Standl., Publ. Field Mus. Nat. Hist., Bot. Ser. 11(5): 172. 1936 =Jacquemontia densiflora Rusby, Bull. Torrey Bot. Club 26: 151. 1899. Tipo: BOLÍVIA. Guanai, 2,000 feet, V-1886, H.H. Rusby 1845 (holótipo NY[NY00319294]!; isótipos NY[NY00319293]!, NY[NY00319295]!, foto MICH!, foto MIN!, foto US!); s.d., M. Bang 2849 (síntipos K! WIS foto!).

Figura 4

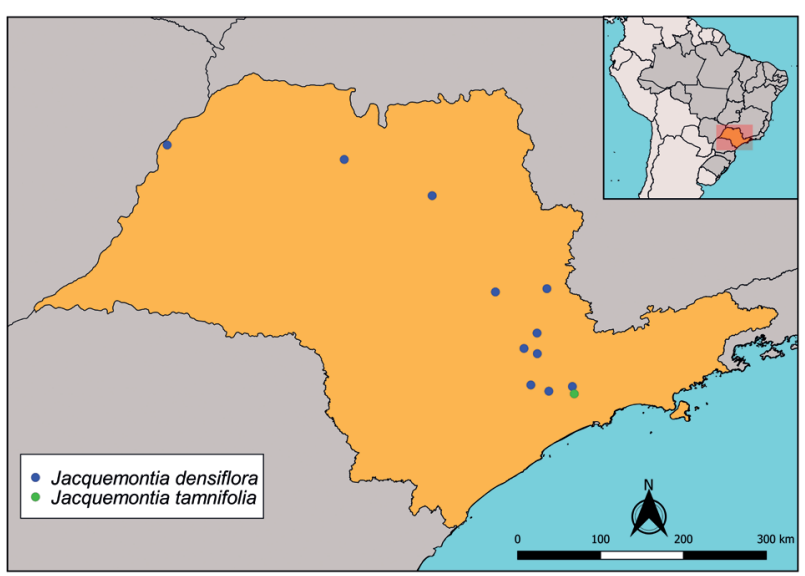

Figura 4. Mapa com a distribuição de Jacquemontia densiflora e Jacquemontia tamnifolia no Estado de São Paulo, Brasil.

Figure 4. Distribution map of Jacquemontia densiflora and Jacquemontia tamnifolia in São Paulo State, Brazil.

O síntipo Warming s.n. (BR0000005792948) foi selecionado como lectótipo de J. violacea var. densiflora, pois é o espécime que mais assemelha-se com a ilustração do protólogo e foi determinado pelo próprio autor.

Jacquemontia violacea var. densiflora é a única variedade da espécie que não foi considerada como sinônimo de $J$. pentanthos (Jacq.) G. Don, pois as demais possuem poucas variações como densidade do indumento, coloração da corola, tamanho do pedúnculo e ápice das folhas.

Jacquemontia densiflora diferencia-se de $J$. pentanthos pela inflorescência mais densa e flores muito menores, com sépalas de até $5 \mathrm{~mm}$ compr. e corola de 6-7 mm compr., enquanto $J$. pentanthos apresenta sépalas com 6-10 mm compr., corola com 1,5-2 cm compr. e inflorescência mais laxa.

Jacquemontia polyantha (Schltdl. \& Cham.) Hallier f.tambémé muito semelhante morfologicamente à $J$. densiflora, porém é mais confundida com $J$. pentanthos devido ao tamanho das flores. Segundo Robertson (1971) e Yang \& Buril (2012) J. polyantha, ocorrente no México, se diferencia de $J$. pentanthos pelas cimeiras mais laxas e sépalas externas ovadas com ápice agudo ou levemente acuminado.

Jacquemontia densiflora ocorre no Equador, Venezuela, Peru, Bolívia, Paraguai, Argentina e Brasil (Austin 1982) nas regiões Nordeste, Sudeste e CentroOeste, em vegetação de Cerrado e Floresta Estacional Semidecídua (Flora do Brasil 2020, em constr.). Coletada com flores e frutos entre abril e maio.

Não se enquadra em nenhum dos critérios propostos por Mamede et al. (2007), portanto foi 
classificada na categoria menos preocupante (LC) no Estado São Paulo.

Material examinado selecionado: BRASIL. São Paulo: Campinas, XI-1995, A.P. Spina 440 (UEC); Indaiatuba, 19-IV-1995, R. Simão-Bianchini 690 et al. (SP); Itapura, IV-1926, J. Venâncio 2221 (R); Itupeva, IV-1995, R. Simão-Bianchini et al. 686 (PMSP, SP, SPF, UEC). Jaboticabal, IV-1997, R. Simão-Bianchini \& E.A. Rodrigues 1052 (SP, PMSP); Mogi Guaçu, V-1993, S. Romaniuc Neto et al. 1368 (SP). Rio Claro, Floresta Estadual Navarro de Andrade, 25-V-1998, L.C. Moura \& V.B. Ziparro 12 (HRCB); São José do Rio Preto, IV-2009, I.M. Silva 66 (IAC). São Paulo, Pirituba, IV-1954, G. Hashimoto 20514 (SP); São Roque, Estação Experimental do Instituto Agronômico de Campinas, 24-IV-1995, L.C. Bernacci et al. 1445 (IAC, SP).

Ilustrações: Meisner (1869, tab. 108) como Jacquemontia violacea var. densiflora; O'Donell (1960b p. 21, fig. 5 A-L) como Jacquemontia rusbyana.

4. Jacquemontia ferruginea Choisy, Mém. Soc. Phys. Genève (8)1: 139. 1838. Tipo: BRASIL. São PaUlo: 1835, P.W. Lund 765 (holótipo foto G!). Convolvulus ferrugineus Steud., in Steudel, Nomencl. Bot., (ed. 3) 2(1): 409. 1840, nom. nud. Jacquemontia ferruginea var. elongata Choisy, in DC. Prodr. Syst Nat. 9: 396. 1845 =Jacquemontia ferruginea var. ambigua Meisn., in Mart., Fl. Bras. 7: 300. 1869, nom. illeg. Tipo: BRASIL. BAHIA: Jacobina, J.S. Blanchet 2629 (lectótipo foto G[00222065]!, aqui designado; isolectótipos foto BR!; foto G[00222064]!, P!); Martii Herbar. Florae Brasil 240 (lectosíntipo foto $\mathrm{BR}$, foto $\mathrm{G}$ !, foto $\mathrm{M}$ !, K!, P!). Jacquemontia grandiflora Meisn. var. grandiflora Meisn., in Mart., Fl. Bras. 7: 300. 1869. Tipo: BRASIL. Martii Herbar. Florae Brasil 240 (lectótipo foto BR[000000583774]!, aqui designado; isolectótipos foto BR[000000583775]!, foto G!, foto M!, K!, P!).

Figuras 3 g-i, 8 a-f, 9

O espécime Blanchet 2629 (G00222065) foi selecionado como lectótipo de Jacquemontia ferruginea var. elongata, por ter sido determinado pelo Choisy e por estar depositado no herbário de Genebra, onde o autor desenvolveu seus estudos. Para $J$. grandiflora está sendo designado como lectótipo um dos tipos depositados no herbário de Bruxelas (BR000000583774), pois possui a determinação de Meisner, além de ser a única exsicata que refere "Claussen" como no protólogo do táxon.
Após sete anos da publicação original de Jacquemontia ferruginea, Choisy (1845) descreveu três variedades para esta espécie (tabela 1): $J$. ferruginea var. ferruginea diferenciada das demais variedades pelo indumento ferrugíneo-tomentoso nos ramos e em ambas as faces das folhas; $J$. ferruginea var. elongata diferenciada pelo indumento alvopubescente e pedúnculos maiores que o tamanho das folhas; e $J$. ferruginea var. rufa pelo indumento avermelhado-tomentoso nos ramos e face abaxial das folhas, face adaxial glabrescente e inflorescência com brácteas muito grandes.

Consideramos $J$. ferruginea var. elongata como sinônimo de $J$. ferruginea, pois os tipos de ambos os táxons examinados apresentam indumento ferrugíneotomentoso e o tamanho do pedúnculo é variável nessa espécie ( 2 a $20 \mathrm{~cm}$ compr). Choisy (1845) referiu dois síntipos para este táxon, no entanto Meisner (1869) considerou esses espécimes como dois novos táxons: o espécime Blanchet 2629 foi reconhecido como $J$. ferruginea var. ambigua Meisn. (nome ilegítimo); e o espécime Martii Herbar 240 como J. grandiflora var. grandiflora Meisn. (sinônimo de $J$. ferruginea).

Jacquemontia ferruginea var. rufa foi elevada à espécie por Hallier (1893), é endêmica de Minas Gerais, muito comum na Serra do Caraça (tabela 1). Jacquemontia rufa é claramente distinguida de $J$. ferruginea pelas folhas oblongas com face adaxial glabra a glabrescente (vs. ovada a lanceolada com face adaxial tomentosa a pubescente) e sépalas glabras, apenas ciliadas ( $v s$. tomentosas a vilosas).

Meisner (1869) também descreveu J. ferruginea var. mucronata, posteriormente reconhecida como sinônimo de J. guyanensis (Aubl.) Meisn. por Hallier (1893). Espécie restrita da região Amazônica, diferencia-se de $J$. ferruginea pelas sépalas todas iguais em tamanho ( $v s$. sépalas externas maiores que as internas), lanceoladas ( $v s$. rômbicas a ovadas), com ápice agudo (vs. ápice acuminado) e indumento mais denso.

Jacquemontia ferruginea é restrita ao território brasileiro, ocorrendo nos Estados da Bahia, Minas Gerais, Rio de Janeiro, São Paulo, Paraná e Santa Catarina. Muito frequente em São Paulo, nas bordas da Floresta Ombrófila, Floresta Estacional Semidecídua e Cerrado, além de áreas antropizadas. Coletada com flores e frutos em todos os meses do ano, com mais frequência entre novembro e maio.

Classificada como menos preocupante (LC) no Estado de São Paulo de acordo com a metodologia de Mamede et al. (2007), pois não se enquadra em nenhum dos critérios utilizados. 
Tabela 1. Atualização nomenclatural das variedades propostas para Jacquemontia ferruginea.

Table 1. Nomenclatural update of the varieties proposed for Jacquemontia ferruginea.

\begin{tabular}{lcc}
\hline Variedades de J. ferruginea & Nome atual & Localidade do tipo \\
\hline J. ferruginea var. ferruginea & J. ferruginea & São Paulo \\
J. ferruginea var. ambigua (nom. illeg.) & J. ferruginea & Bahia \\
J. ferruginea var. elongata & J. ferruginea & Bahia \\
J. ferruginea var. rufa & J. rufa & Minas Gerais \\
J. ferruginea var. mucronata & J. guyanensis & Região Amazônica \\
\hline
\end{tabular}

Material examinado selecionado: BRASIL. São PaUlo: Alumínio, 10-XII-2012, A.M.G.A. Tozzi et al. 306(UEC); Angatuba, Estação Ecológica de Angatuba, 2314'59"S, 48¹1'53"W, 18-XII-2012, M. Pastore et al. 207 (SP, ESA); Araçoiaba da Serra, 2317'26"S, 47²2'58"W, 18-XII-2012, M. Pastore et al. 213 (SP, ICN, PMSP); Araraquara, 30-XI-1967, H.M. Souza s.n. (IAC 20281); Bofete, Fazenda Santa Terezinha, 12-II-1993, J.C. Nordi 4 (BOTU); Entre São Carlos e Descalvado, Fazenda Graciosa, 3-II-1966, J.B. Feliciano 28 (SP); Iguape, Estação Ecológica JureiaItatins, Trilha do Imperador, 6-I-2000, R.J.F. Garcia et al. 1867 (SP, PMSP); Iperó, Floresta Nacional de Ipanema, 2315'53"S, 47²1'33"W, 19-XII-2012, M. Pastore et al. 210 (SP, SPSF); Itanhaém, Ilha da Queimada Grande, 23-I-1997, G.O. Joaquim Jr. et al. 110 (ESA); Itapetininga, II-1946, J.T. Lima et al. s.n. (RB55450); Itapeva, Estação Experimental de Itapeva, 240.'30"S, 4904'05"W, 25-VI-2009, C.R. Lima et al. 208 (SPSF); Itararé, Bom Sucesso de

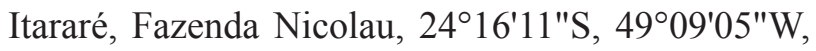
10-XII-1997, S.I. Elias et al. 30 (SP, ESA); Jundiaí, Reserva Biológica Municipal da Serra do Japi, 2313'55"S, 4656'28"W, 18-II-2008, J.A. Lombardi et al. 7255 (HRCB); Juquitiba, Estrada da Palestina, BR 116, km 4, 2358'32"S, 4705'25"W, 26-XII2008, R. T. Polisel 968 (SPSF); Mogi das Cruzes, Vila São Geraldo, 10-II-1938, G. Hashimoto \& A. Gehrt 261 (SP); Peruíbe, Estação Ecológica Juréia-Itatins, Trilha do Imperador, ca. 1,5 Km S do rio Verde, 28V-1996, L.P. de Queiroz et al. 4520 (SP); Piracicaba, Mata do Bairo Recreio, 22³4'13.3"S, 4704'30.4"W, 4-I-1994, K.D. Barreto et al. 1706 (SP, ESA); Queluz, SP 54, estrada do hotel Ninho do Condor até a cidade, 24-V-1996, I. Koch et al. 525 (SP, SPF, UEC); Rio Claro, 25-XI-2005, M.F.C. Barbosa s.n. (HRCB42821); Santa Cruz das Palmeiras, 3-XII-1990, B. Essor (IAC31928); Santa Isabel, Funil, 10-II-1991, Hashimoto 20502 (SP); Santa Rita do Passa Quatro,
Pé Gigante, 2136'44"S, 47³4'41"W, 3-I-1996, M.A. Batalha 1042 (SP); Santo Antônio do Pinhal, Pico Agudo, 2249'38"S, 45³9'45"W, 14-11-2004, S.E. Martins 885 (SP); Santos, Ilha de Alcatrazes, X-1920, H. Luederwaldt Fonseca 10910 (SP); São Bernardo do Campo, Beira da Rodovia Imigrantes km 34, 26-XII-2012, M. Pastore \& R.M. Brito 215 (SP, PMSP); São José do Barreiro, Parque Nacional da Serra da Bocaina, próximo a sede do Parque, na parte baixa, 21-I-2013, M. Pastore et al. 229 (SP, BOTU, ESA, IAC, PMSP, SJRP); São José dos Campos, Reserva Florestal da Boa Vista, 14-XII-1987, A.F. Silva 1587 (UEC); São Paulo, Parque Estadual Fontes do Ipiranga, Rampa de acesso ao Instituto de Botânica., 2338'08"S, 46³6'48"W, 8-III-2006, B.L.P. Villagra 36 (SP); Sorocaba, Votorantin, 17-IV-1995, R.Simão-Bianchini et al. 651 (SP, SPF, UEC, PMSP); Tatuí, Continuação da Rua Adolfo Assunção, próximo ao trilho do trêm, 28-X-2012, M. Pastore 199 (SP, HRSB); Taubaté, Estrada entre Taubaté e Campos do Jordão, 2301'35"S, 45³3'19"W, 22-XI-2006, C.V. Silva 55 (SP); Teodoro Sampaio, Parque Estadual do Morro do Diabo, 21-VI-1994, O.T. Aguiar 464 (SP); Tietê, 10-IX-1969, C. Aranha s.n. (IAC); Votorantim, A.M.G.A. Tozzi et al. 299 (UEC).

Ilustrações: Ferreira (2013, p. 294, fig. 2 A-B, sépalas e gineceu).

5. Jacquemontia frankeana (Schltdl.) M. Pastore \& Sim.-Bianch., Phytotaxa 221(2): 194. 2015 三 Convolvulus frankeanus Schltdl., Linnaea 9: 513. 1834. Tipo: BRASIL. RIO DE JANEIRO: M.R. Franks.n. (holótipo foto HAL!). Jacquemontia uleana Hallier f., Jahrb. Hamburg. Wiss. Anst. 16, Beiheft 3: 31. 1899. Tipo: BRASIL. Rio DE JANEIRO: Restinga do Arpoador, E.H.G. Ule 4388 (holótipo B, destruído, foto $\mathrm{F}$ ! lectótipo R!, aqui designado; isolectótipos foto LIL!, MO).

Figuras 3 j-1, 8 g-h, 12 
Como o holótipo de J. uleana, depositado no herbário de Berlim (B), foi destruído durante a Segunda Guerra Mundial, a duplicata depositada no herbário do Museu Nacional (R) foi selecionada como lectótipo.

Jacquemontia frankeana é muito semelhante morfologicamente à $J$. holosericea, mas a primeira possui ramos e folhas pubérulos a glabrescentes com tricomas de raios adpressos na face abaxial das folhas e a segunda possui ramos e folhas tomentosos a pubescentes com tricomas de raios eretos na face abaxial das folhas. Há alguns indivíduos intermediários entre estas duas espécies o que pode ser uma evidência de hibridação. Um exemplo é observado no espécime J.C. Braidotti 117 (SP) que apresenta ramos e folhas glabrescentes como em $J$. frankeana, entretanto os tricomas são eretos como em $J$. holosericea. São necessários ainda mais estudos sobre esses táxons, como genética de populações, biologia floral e biologia molecular para melhor entender este complexo. Ambas espécies são comuns nas regiões litorâneas de São Paulo e Rio de Janeiro.

Jacquemontia frankeana é restrita ao território brasileiro, ocorre nos Estados do Rio de Janeiro, São Paulo, Paraná e Santa Catarina, em Restinga e bordas da Floresta Ombrófila Densa. Coletada com flores e frutos de setembro a junho.

Considerada como menos preocupante (LC) de acordo com a metodologia de Mamede et al. (2007), pois enquadra-se apenas no critério 9 por ser restrita a um tipo de vegetação.

Material examinado selecionado: BRASIL. São Paulo. Cananéia, Parque Estadual da Ilha do Cardoso, Próximo à Praia Itacuruça, 11-III-2013, M. Pastore \& A.A. Lemos 276 (SP); Eldorado, Fragmento de mata, próximo à Rua Silvério Pupo, 13-X-2013, E.P. Fortes 2 (SP); Iguape, 30-V-1986, E.L.M. Catharino 766 (SP, ESA); Ilhabela, Parque Estadual de Ilhabela, Sul da Ilha de São Sebastião, 12-II-2013, M. Pastore \& R.M. Brito 232 (SP); Iporanga, Caminho para a cachoeira Queda de Meu Deus, 27-XII-2002, $R$. Simão-Bianchini 1530 (SP); Juquiá, em direção a Tapiraí, 9-IX-1994, C.A. Monteiro et al. 23 (SP, SPF, PMSP, ESA, HRCB, UEC); Pariquera-Açu, Rodovia de acesso à BR 116, km 4, 11-I-2005, R.G. Udulutsch A. \& Udulutsch-Filho 2614 (HRCB); Pedro de Toledo, Rodovia Padre Manoel da Nóbrega, próximo a entrada da cidade, $2^{\circ} 16^{\prime} 29^{\prime \prime} \mathrm{S}, 47^{\circ} 36^{\prime} 58^{\prime \prime} \mathrm{W}, 10-\mathrm{III}-2013$, M. Pastore 275 (SP); São Sebastião, 27-XII-1971, J. Mattos N. Mattos 15729 (SP); Tapiraí, Reserva
Particular da Votorantim, Trilha Casa das Pedras, 2409'55"S, 47³3'36"W, 28-III-2013, C. Delfine et al. 866 (SP, ESA); Ubatuba, Parque Estadual da Ilha Anchieta, Trilha do Saco Grande, 4-I-2013, M. Pastore \& R.M. Brito 221 (SP).

Ilustrações: como Jacquemontia holosericea em Simão-Bianchini (2005, p. 146, fig. 15-17); como Jacquemontia uleana em Ferreira (2013, p. 295, fig. 3 G-H).

6. Jacquemontia gabrielii (Choisy) Buril, Phytologia 97(3): 221. 2015. झ Ipomoea gabrielii Choisy in De Candolle, Prodr. 9: 378. 1845. Tipo: GUIANA FRANCESA. Cayenne, Gabriel s.n. (holotype G). Jacquemontia ciliata Sandwith, Bull. Misc. Inform. Kew 4: 156. 1930. Tipo:TRINIDAD. On the outskirts of Irois Forest, 25-I-1925, W.E. Broadway 6178 (holótipo K[000613049]!; isótipos foto BM!, K!, foto $\mathrm{MO}$ !, foto $\mathrm{S}$ !).

Figuras 2, 5 g-m, 6 a-c

Jacquemontia gabrielii pode ser confundida principalmente com $J$. holosericea, J. frankeana e $J$. velutina. As duas primeiras se diferenciam especialmente pelas sépalas externas menores que as internas ( $v s$. todas iguais a subiguais), tricomas estrelados 4-5-radiados (vs. 3-radiados) e grãos de pólen tricolpados (vs. pantocolpados). Enquanto $J$. velutina diferencia-se pelos ramos e folhas velutinos (vs. hirsutos), tricomas estrelados 3-radiados com raios iguais a subiguais em ambas as faces da folha (v.s. 3-radiados com o raio central mais longos que os laterais na face adaxial das folhas), sépalas com 4-6 mm compr., pubescentes (vs. 6-8 mm compr., glabras ou apenas ciliadas) e áreas mesopétalas da corola completamente glabras (vs. pilosas apenas no ápice).

Espécie encontrada no México, Costa Rica, Panamá, Colômbia, Trindade e Tobago, Guiana Francesa, Venezuela, Equador, Peru e Brasil (Robertson 1971; Austin \& Cavalcante 1982) nos Estados do Pará e Rondônia, com disjunção em São Paulo e Rio de Janeiro. Na área de estudo foi encontrada apenas em restingas do Parque Estadual Serra do Mar Núcleo Picinguaba. Coletada com flores de outubro a maio e com frutos de fevereiro a maio.

Para a avaliação de conservação estadual seguindo os critérios propostos por Mamede et al. (2007), J. gabrielii foi classificada como vulnerável (VU) no Estado de São Paulo. Enquadra-se nos critérios 3, 5 e 9 , referentes respectivamente à distribuição restrita 


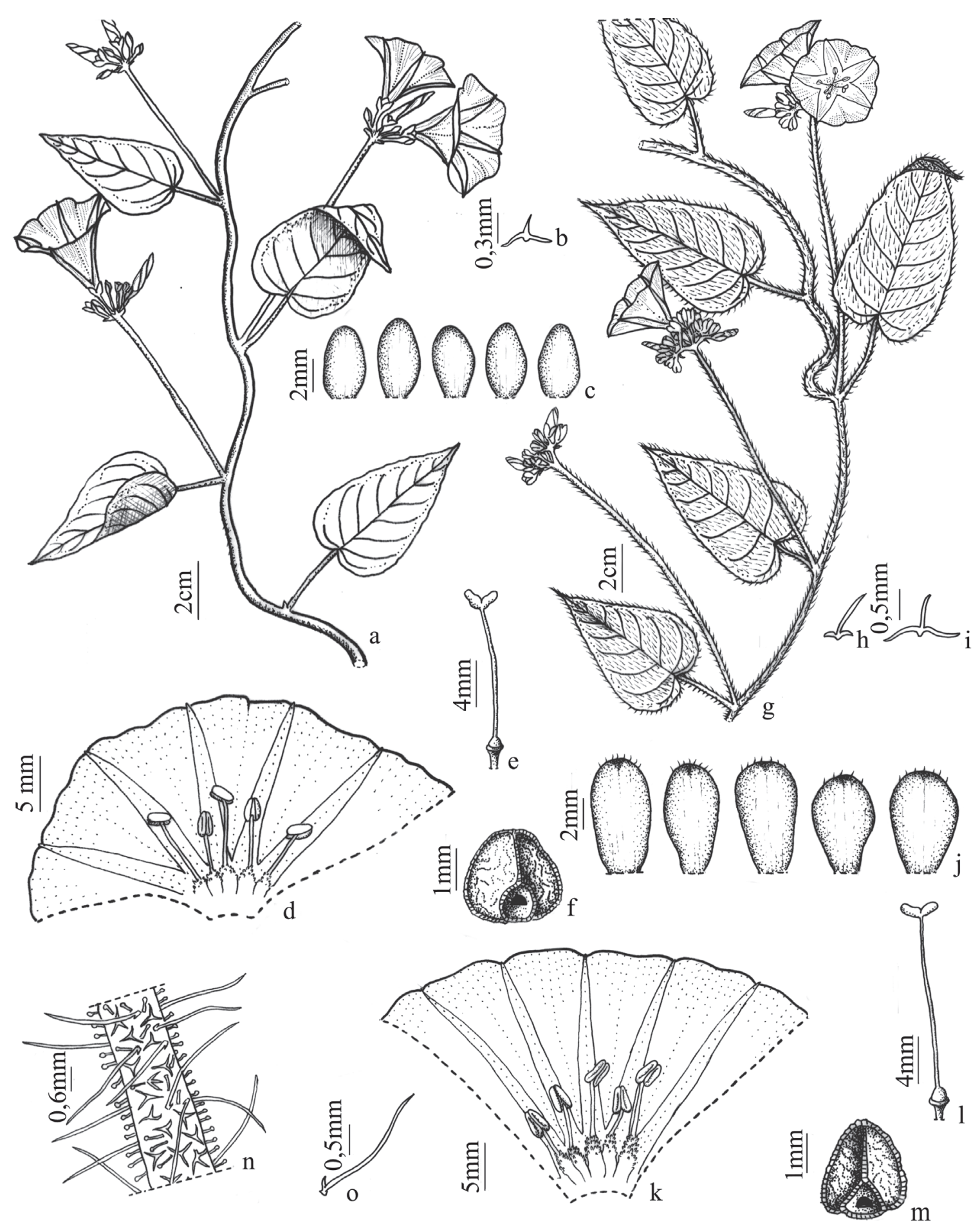

Figura 5. a-f. Jacquemontia blanchetii. a. Hábito. b. Tricoma da superfície abaxial da folha. c. Sépalas ordenadas da mais externa para a mais interna. d. Corola em corte longitudinal. e. Gineceu. f. Semente (M. Pastore \& S.A. Adachi 271). g-m. Jacquemontia gabrielii. g. Hábito. h. Tricoma da superfície adaxial da folha. i. Tricoma da face abaxial da folha. j. Sépalas ordenadas da mais externa para a mais interna. k. Corola em corte longitudinal. 1. Gineceu. m. Semente (M. Pastore \& F.C. Brito 225). n-o. Jacquemontia sphaerostigma. n. Tipo de tricomas no ramo. o. Tricoma forcado (E.L.M. Catharino et al. 1862).

Figure 5. a-f. Jacquemontia blanchetii. a. Habit. b. Trichome of the abaxial surface of the leaf. c. Sepals arranged from the outermost to the innermost. d. Corolla in a longitudinal cut. e. Gynoecium. f. Seed (M. Pastore \& S.A. Adachi 271). g-m. Jacquemontia gabrielii. g. Habit. h. Trichome of the adaxial surface of the leaf. i. Trichome of the abaxial surface of the leaf. j. Sepals arranged from the outermost to the innermost. k. Corolla in a longitudinal cut. 1. Gynoecium. m. Seed (M. Pastore \& F.C. Brito 225). n-o. Jacquemontia sphaerostigma. n. Type of trichomes on the stem. o. Furcated trichome (E.L.M. Catharino et al. 1862). 

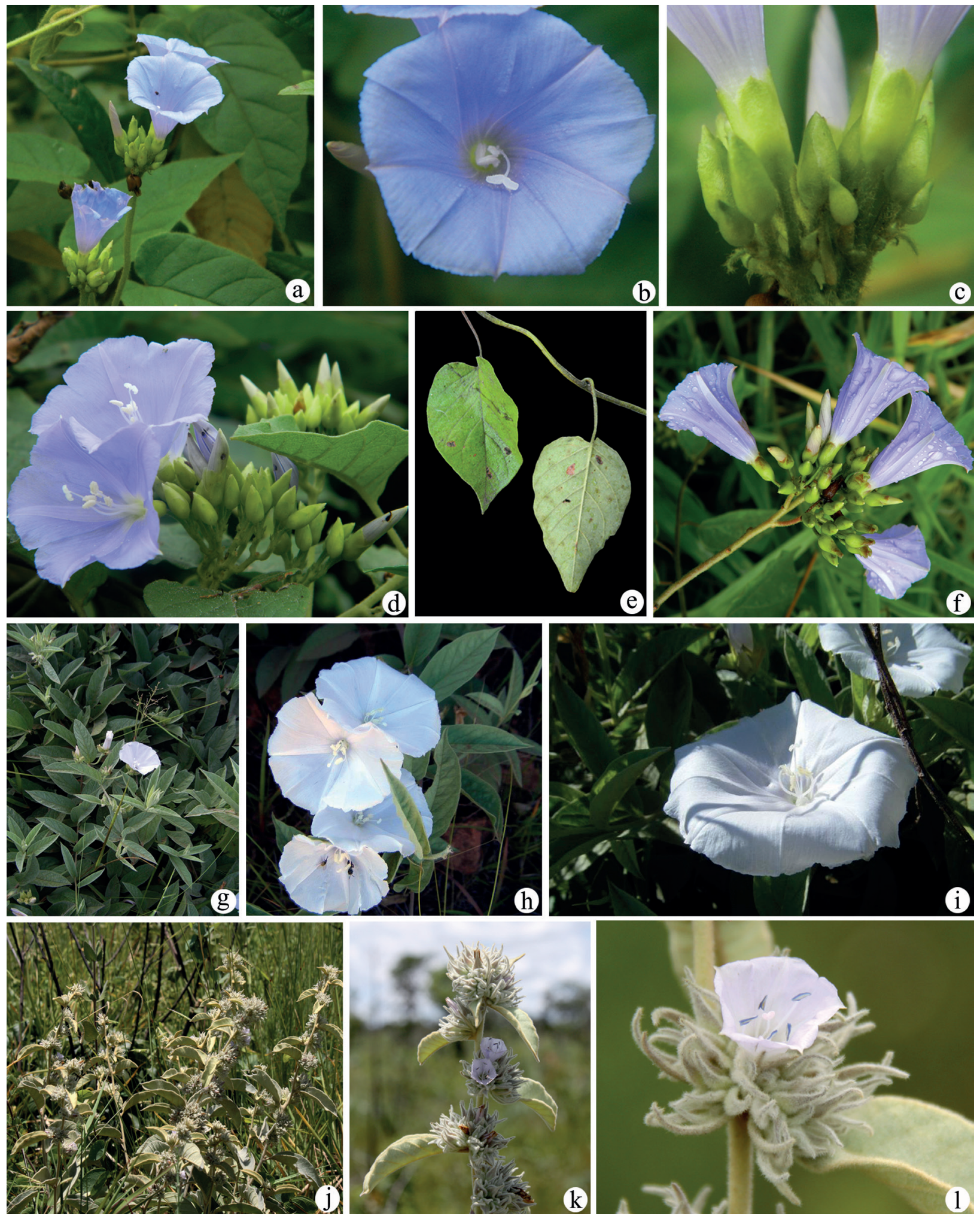

Figura 6. a-c. Jacquemontia gabrielii. a. Ramo com flores e folhas. b. Flor em vista frontal. c. Detalhe dos cálices. d-f. Jacquemontia holosericea. d. Ramo com flores e folhas. e. Folhas. f. Inflorescência. g-i. Jacquemontia selloi. g. Hábito. h. Inflorescência. i. Flor em vista frontal. j-1. Jacquemontia sphaerocephala. j. Hábito. k. Inflorescência. 1. Detalhe da flor. Fotos g-i P.P.A. Ferreira. j-1. H. Moreira.

Figure 6. a-c. Jacquemontia gabrielii. a. Stem with flowers and leaves. b. Flower in front view. c. Detail of the calyxes. d-f. Jacquemontia holosericea. d. Stem with flowers and leaves. e. Leaves. f. Inflorescence. g-i. Jacquemontia selloi. g. Habit. h. Inflorescence. i. Flower in front view. j-1. Jacquemontia sphaerocephala. j. Habit. k. Inflorescence. 1. Detail of the flower. Fotos g-i P.P.A. Ferreira. j-1. H. Moreira. 
(encontrada no Estado em apenas uma quadrícula de $1^{\circ} \times 1^{\circ}$ ), exclusiva em unidade de conservação e restrita em um tipo de vegetação.

Material examinado selecionado: BRASIL. SÃo PAUlo: Ubatuba, Parque Estadual da Serra do Mar Núcleo Picinguaba, 2321'8.09"S, 4451'13.29"W, 7-I-2013, M. Pastore \& F.C. Brito 225 (SP, SPSF).

Material examinado adicional: BRASIL. Rio DE Janeiro: Niterói, Parque Estadual da Serra de Tiririca, 26-IV-2001, M.C.F. Santos et al. 269 (RB); Paraty, 4-VIII-1994, R. Marquete 1919 (RB); Rio Bonito, 21-XII-1975, P. Laclette 1 (R); Silva Jardim, Reserva Biológica do Poço das Antas, 23-II-1994, A. Piratininga et al. 33 (MBM, RB, SP).

7. Jacquemontia glabrescens (Meisn.) M. Pastore \& Sim.-Bianch. Phytotaxa 221(2): 195. 2015. इ Jacquemontia grandiflora Meisn. var. glabrescens Meisn., in Mart., F1. Bras. 7: 300. 1869. Tipo: BRASIL. Minas Gerais: Caldas, A. Regnell III 201 (lectótipo foto BR[000000583781]!, designado por Pastore \& Bianchini [2015]; isolectótipos foto BR[00000583717]!, foto BR[00000583718]!,P! foto $\mathrm{S} !)$.

Figura 7

Meisner (1869) diferenciou J. grandiflora de $J$. ferruginea pela inflorescência capituliforme contraída ( $v s$. inflorescência corimbiforme), entretanto consideramos que os tipos de ambos os táxons apresentam inflorescências corimbiformes. Portanto, J. grandiflora está sendo considerada como sinônimo de $J$. ferruginea, assim como propôs Hallier (1899). Enquanto J. grandiflora var. glabrescens foi considerada como uma espécie distinta e seu status foi elevado (Pastore \& SimãoBianchini 2015). Jacquemontia glabrescens se distingue de $J$. ferruginea pelas folhas com ápice acuminado ( $v s$. obtuso a agudo), sépalas glabras a glabrescentes ( $v s$. tomentosas a vilosas), as externas com 9-13 × 4,5-6 mm (vs. 7-11 × 2,5-5 mm) e as internas com 7-9 $\times 5-5,5 \mathrm{~mm}$ (vs. 5-7 $\times 2-3 \mathrm{~mm}$ ).

Endêmica do Brasil, ocorre em Minas Gerais, Rio de Janeiro, São Paulo e Paraná, em bordas de Floresta Ombrófila e Floresta Estacional Semidecídua. Coletada com flores e frutos de dezembro a fevereiro.

Espécie quase ameaçada (NT) no Estado São Paulo, de acordo com a metodologia de Mamede et al. (2007), pois enquadra-se nos critérios 4 e 9 , referentes respectivamente à ocorrência desconhecida em unidades de conservação e exclusiva em um tipo de vegetação.
Material examinado selecionado: BRASIL. SÃo Paulo: Campos do Jordão, 5-II-1937, P.C. Porto 3236 (RB); Cruzeiro, Pico dos Martins, 3-XII-1997, G. Hashimoto 17392 (GHSP, SP); São Bento do Sapucaí, Pedra do Baú, 18-I-1997, S. Oki 20498 (GHSP, SP); São Francisco, I-1897, Loefgren CGG3507 (SP).

Material examinado adicional: BRASIL. MinAS GeraIs: Caldas, 21-I-1919, F.C. Hoehne s.n. (SP2896); Itamonte, Serra Fina, Hotel Alpina, 19-II-2004, L.D. Meireles et al. 1517 (SP, UEC). Passa Quatro, Pico do Itaguaré, 21-III-2006, L.D. Meireles \& J.A. Nunes 2118 (SP, UEC). Poços de Caldas, I-1919, F.C. Hoehne s.n. (SP2845). PARANÁ: Chopinzinho, Rio Iguaçu, Santo Santiago, 11-IV-1975, G. Hatscbach 36629 (MBM, SP). Rio DE JANEIRO: Itatiaia, Parque Nacional do Itatiaia, 22-IV-2008, L.A.F. Santos Filho et al. 43 (SP, RB).

8. Jacquemontia holosericea (Weinm.) O'Donell, Lilloa 26: 357. 1953. इIpomoea holosericea Weinm., Syll. Plant. Nov. 2: 17. 1828. Tipo: BRASIL. Rio DE JANEIRO: 1839, G. Schüch s.n. (neótipo P[03867913]!, aqui designado; isoneótipos P[03867912]!, P[03867914]!, GH). Jacquemontia menispermoides Choisy, Mém. Soc. Phys. Genève (8)1: 63. 1838. Tipo: BRASIL. Rio DE JANEIRO: $C$. Gaudichaud 32 (lectótipo foto G!, designado por Buril \& Staples [2017]); C. Gaudichaud 566(lectosíntipo P!); Graham s.n., (lectosíntipo K!). Ipomoea crotonifolia Gardner, Lond. Journ. Bot. 1: 180. 1842. Tipo: BRASIL. Rio DE JANEIRO: “Corcovado, and at Rio Comprido", XI-1836, G. Gardner 80 (lectótipo $\mathrm{K}$ [000945292]!, aqui designado; isolectótipos

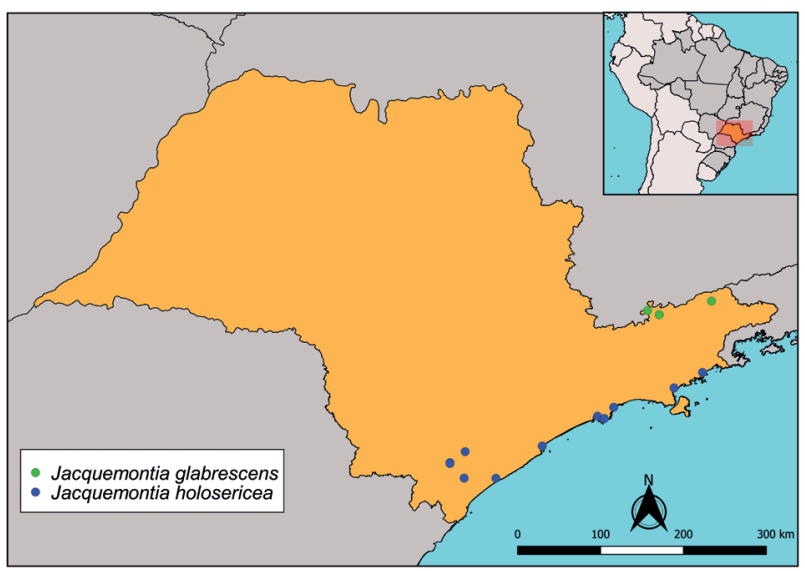

Figura 7. Mapa com a distribuição de Jacquemontia glabrescens e Jacquemontia holosericea no Estado de São Paulo, Brazil.

Figure 7. Distribution map of Jacquemontia glabrescens and Jacquemontia holosericea in São Paulo State, Brazil. 


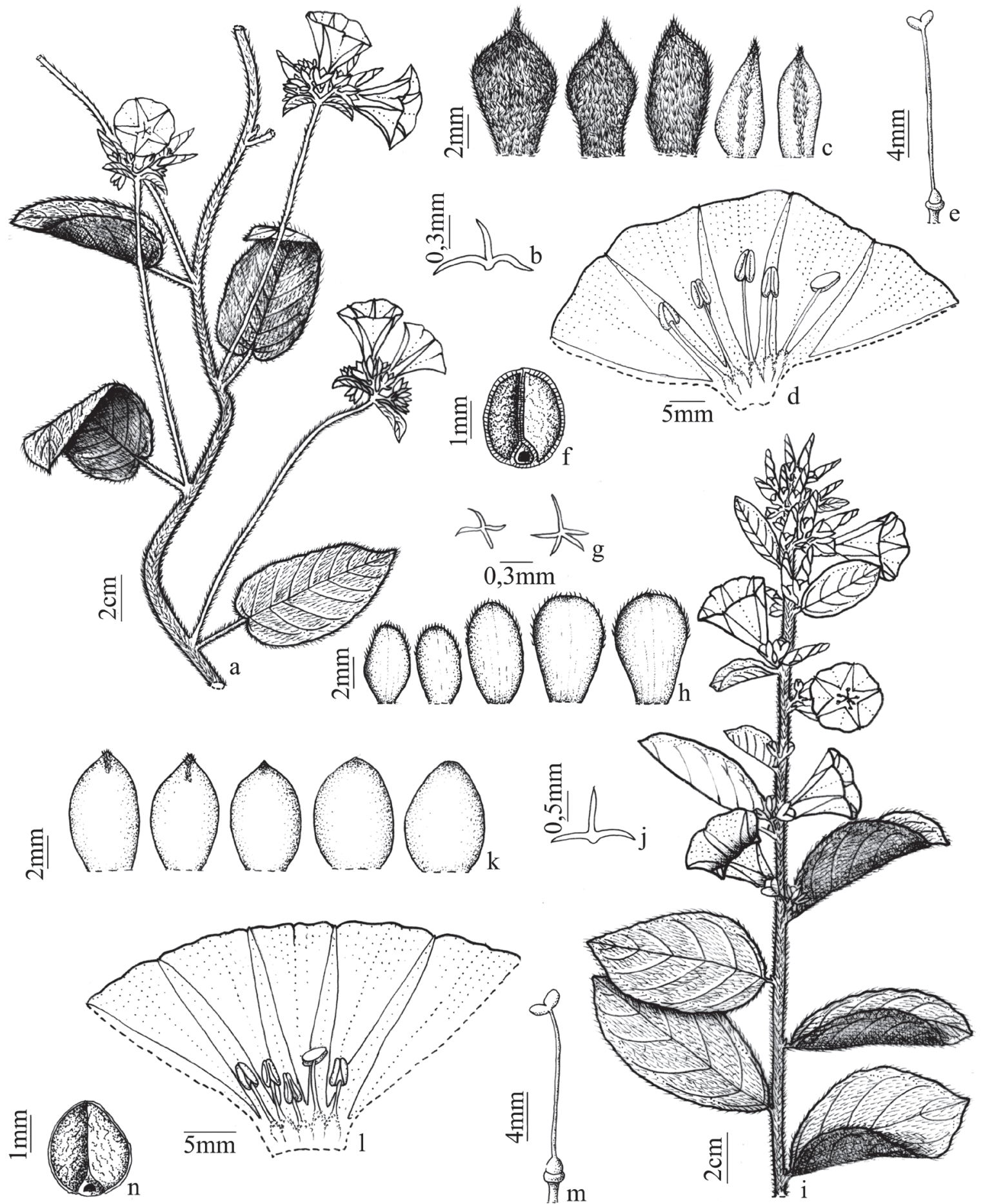

Figura 8. a-f. Jacquemontia ferruginea. a. Hábito. b. Tricoma da superfície adaxial da folha. c. Sépalas ordenadas da mais externa para a mais interna. d. Corola em corte longitudinal. e. Gineceu. f. Semente (M. Pastore 199). g-h. Jacquemontia frankeana. g. Tricomas da superfíce abaxial da folha. h. Sépalas ordenadas da mais externa para a mais interna (M. Pastore \& A.A. Lemos 276). i-n. Jacquemontia spiciflora. i. Hábito. j. Tricoma da superfície abaxial da folha. k. Sépalas ordenadas da mais externa para a mais interna. 1. Corola em corte longitudinal. m. Gineceu. n. Semente (M. Pastore et al. 237).

Figure 8. a-f. Jacquemontia ferruginea. a. Habit. b. Trichome of the adaxial surface of the leaf. c. Sepals arranged from the outermost to the innermost. d. Corolla in a longitudinal cut. e. Gynoecium. f. Seed. (M. Pastore 199). g-h. Jacquemontia frankeana. g. Trichomes of the abaxial surface of the leaf. h. Sepals arranged from the outermost to the innermost (M. Pastore \& A.A. Lemos 276). i-n. Jacquemontia spiciflora. i. Habit. j. Trichome of the abaxial surface of the leaf. k. Sepals arranged from the outermost to the innermost. 1. Corolla in a longitudinal cut. m. Gynoecium. n. Seed (M. Pastore et al. 237). 


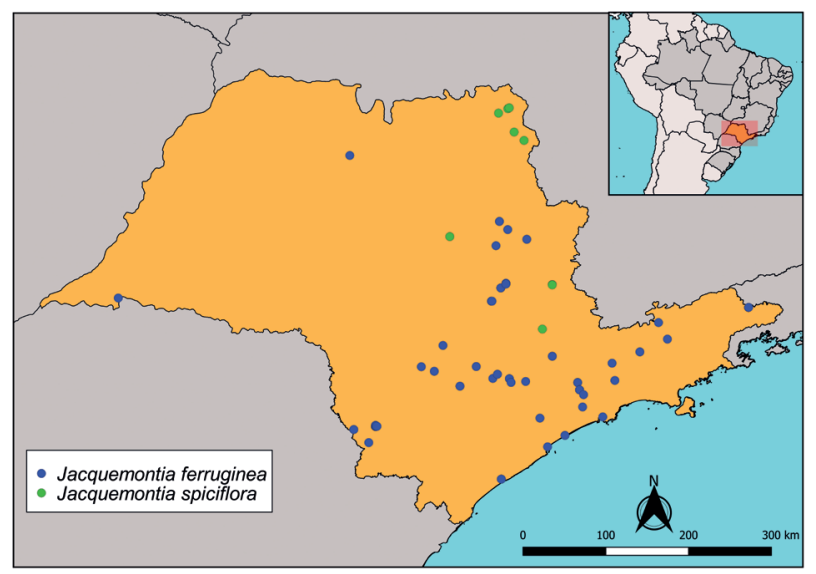

Figura 9. Mapa com a distribuição de Jacquemontia ferruginea e Jacquemontia spiciflora no Estado de São Paulo, Brasil.

Figure 9. Distribution map of Jacquemontia ferruginea and Jacquemontia spiciflora in São Paulo State, Brazil.

K[000945300]!, NY!, P!). Jacquemontia menispermoides var. canescens Meisn., in Mart., Fl. Bras. 7: 295 1869. Tipos: BRASIL. Rio DE JANEIRO: 1867, A.F.M. Glaziou 690 (lectótipo foto BR[0000005793921]!, designadopor Buril \& Staples [2017]; isolectótipos foto BR[0000005793594]!, foto BR[0000005792238]!); Warming s.n. (lectosíntipo C).

Figuras 6 d-f, 7

Nenhum material-tipo ou ilustração para Ipomoea holosericea foram apresentados no protólogo, a espécie foi descrita com base a indivíduos cultivados de sementes provenientes do Brasil, e O'Donell (1953) também não designou o neótipo. Portanto selecionamos o material Vauthier 255 (P) como neótipo, o qual está de acordo com a descrição original, encontra-se em bom estado, com a presença de flores completas, além de sua duplicata $(\mathrm{GH})$ ter sido citada por O'Donell (1953).

Para Ipomoea crotonifolia selecionamos como lectótipo o material Gardner 80 (K000945292), pois é o único que apresenta a descrição de localidade como no protólogo, além das coletas de Gardner realizadas no Brasil terem sido depositadas no herbário do Kew Garden.

Jacquemontia holosericea mais assemelha-se a $J$. frankena, e as diferenças entre elas são discutidas sob a última espécie.

Jacquemontia holosericea é restrita ao território brasileiro, ocorre nos Estados de Minas Gerais, Espírito Santo, Rio de Janeiro e São Paulo, em bordas da Floresta Ombrófila Densa e Restinga, muito frequente em áreas costeiras. Coletada com flores de setembro a abril e com frutos de janeiro a abril.

De acordo com a metodologia de Mamede et al. (2007) a espécie foi classificada como menos preocupante (LC) no Estado São Paulo, pois enquadra-se apenas no critério 9, ou seja, restrita a um tipo de vegetação.

Material examinado selecionado: BRASIL. São PAUlo: Bertioga, Praia de São Lourenço, 30-III-1997, R. SimãoBianchini \& S. Bianchini 1043 (SP); Caraguatatuba, perto da praia, 15-I-2013, R. Simão-Bianchini S. Bianchini 1873 (SP, BOTU, IAC, PMSP, SJRP); Eldorado, próximo ao portal da cidade de Eldorado, 2431'48"S, 4806'28"W, 11-X-2012, M. Pastore et al. 198 (SP, UEC); Guarujá, beira da Rodovia Rio-Santos, 27-12-2012, M. Pastore R.M. Brito 216 (SP, SPSF); Iguape, Estrada Pariquera-Mirim para Iguape, 15-II1995, H.F. Leitão-Filho et al. 33183 (UEC); PariqueraAçu, Beira da Rodovia SP-222 (José Redis), km 108, 2442'22"S, 4756'16"W, 11-10-2012, M. Pastore et al. 197 (SP, ESA, ICN); Peruíbe, Juréia, 10-X-1988, R. Simão-Bianchini 50 (SP, SPF); Sete Barras, Estrada Sete Barras p/ São Miguel Arcanjo, 13-II-1995, H.F. Leitão-Filho et al. 32815 (UEC).

Ilustrações: como Jacquemontia menispermoides em Meisner (1869, t. 107).

9. Jacquemontia selloi (Meisn.) Hallier f., Bot. Jahrb. Syst. 16:543. 1893. EIpomoea selloi Meisn., in Mart., F1. Bras. 7: 271. 1869. Tipo: BRASIL. Austral, $F$. Sellow s.n. (B, F foto!). Ipomoea selloi var. rufescens Meisn., in Mart., Fl.Bras. 7:271.1869. Tipo: BRASIL. Minas GERAIS: Uberaba, 1867, A. Regnell III 197(lectótipo foto BR[BR0000005306084]!, aqui designado); SÃoPaUlo: RioPardo, 1826, L. Riedel606 (lectosíntipoNY!);BOLÍVIA. SANTACRUz: Chiquitos, 1842, A. D'Orbigny 939 (lectosíntipo foto BR!). Jacquemontia loefgrenii Hoehne, Secc. Bot. 1(6): 55, pl. 11. 1922. Tipo: BRASIL. São PAULO: Itapetininga, 15-IX-1887, Loefgren CGG120 (holótipo SP!; isótipo LIL!, foto C!). Aniseia minor Pilg., Revista Mus. Paul. Univ. São Paulo 13: 1252. 1923. Tipo: BRASIL. Mato Grosso: Bom Jardim, São Luiz de Cáceres, VIII-1908, F.C. Hoehne 239 (síntipo B destruído [empréstimo do SP); Quilombo, Cáceres, IX-1908, F.C. Hoehne 335 e F.C. Hoehne 350 (síntipos B destruídos[empréstimosdoSP]). Brasil,MatoGrosso, Porto Alegre do Norte, 13-X-1938, J.E. Rombouts 284 (neótipo SP!, aqui designado; isoneótipo IAC!).

Figuras. 6 g-i, 11 
O espécime Regnell III 197 (BR0000005306084) foi designado como lectótipo de J. selloi var. rufescens, pois está em bom estado e trata-se do mais completo entre os síntipos.

Os síntipos de Aniseia minor, pertencentes ao herbário SP foram emprestados ao herbário B para o Dr. Pilger (Hoehne 1922), entretanto esses materiais nunca foram devolvidos e foram perdidos durante a Segunda Guerra Mundial. Portanto, designamos o material Rombouts 284 (SP) como neótipo, pois também foi coletado em Mato Grosso e está em ótimo estado de conservação, incluindo flores completas. Não foi possível designar um lectótipo para este táxon, mas Buril \& Staples (2017) recentemente designaram o síntipo Hoehne 350 como lectótipo de Aniseia minor, pois não notaram que este material foi perdido.

Jacquemontia selloi é facilmente reconhecida pelo hábito subarbustivo, frequentemente com alguns ramos escandentes, folhas elípticas a oblanceoladas, base cuneada a atenuada, três a quatro pares de nervuras secundárias, sépalas externas evidentemente maiores que as internas e ovadas.

Esta espécie também já foi reconhecida como Aniseia minor, pois antigamente as espécies de Convolvulaceae com sépalas externas ampliadas, eram consideradas no gênero Aniseia Choisy. Atualmente Aniseia é formado por apenas três espécies, já que as outras foram transferidas para gêneros como Calystegia, Convolvulus, Ipomoea L., Merremia Dennst. ex Endl. e Jacquemontia (Austin 1999, AthiêSouza et al. 2017).

Encontrada na Bolívia, Paraguai, Argentina e Brasil (O’Donell 1960b; Wood et al. 2014) em áreas de campo das Regiões Centro-Oeste, Sudeste e Sul (Flora do Brasil 2020, em constr.). Coletada com flores de outubro a dezembro.

Classificada como menos preocupante (LC) no Estado São Paulo pela metodologia de Mamede et al. (2007), entretanto esta espécie pode vir a ser ameaçada, pois a maioria dos seus registros em São Paulo é anterior a 50 anos e sua ocorrência é restrita em áreas de campo do Cerrado, bioma extremamente ameaçado no Estado.

Material examinado selecionado: BRASIL. São Paulo: Botucatu, 25-IX-1986, L.R.H. Bicudo 1492 (BOTU); Capão Bonito, XII-1949, J. Vidal 330 (R); Itapetininga, estrada para Angatuba, 29-XI-1959, S.M. Campos 19 (SP); Itapeva, X-1950, J. Vidal 375 (R); Pedregulho, Parque Estadual Furnas do Bom Jesus, próxima ao encontro dos córregos Pedregulho e Bom
Jesus, 20¹1'50"S, 47²5'10"W, 2-XI-2003, D. Sasaki et al. 817 (SPF); Tatuí, 1-XII-1936, F.C. Hoehne A. \& Gehrt s.n. (SP37047).

Material examinado adicional: BRASIL. MATO Grosso: Porto Alegre do Norte, X-1938, J.E. Rombouts 284 (SP, IAC). MAto Grosso do Sul: Bonito, $15 \mathrm{~km}$ Sul de Bonito na MS-178, próximo a Fazenda São Carlos, X-1995, A. Pott et al. 7460 (SP, CPAC); Campo Grande, caminho para Capão Bonito, IX-1936, W. Archer \& A. Geht 36348 (SP). Minas Gerais: Caeté, Serra da Piedade, 11-I-1996, V.C. Souza 10096 (ESA). PAranÁ: Jaguariaíva, Parque Estadual do Cerrado, X-2000, V. Linsingen 573 (SP). SANTA Catarina: Abelardo Luz, 15-XI-1964, L.B. Smith \& R.M. Klein 13300 (R).

Ilustrações: O’Donell (1960a, p. 23, fig. 6 A-H); Ferreira (2013, p. 295, fig 3 A-D).

10. Jacquemontia sphaerocephala Meisn., in Mart., Fl. Bras. 7: 306. 1869. E Thyella sphaerocephala (Meisn.) House, Bull. Torrey Bot. Club 33:314. 1906. Tipo: BRASIL. Minas Gerais: Paracatu, "in campis siccis petrosis", 1834, L. Riedel s.n. (holótipo foto LE!; isótipo NY!).Jacquemontia acrocephala Meisn., in Mart., Fl. Bras. 7:306. 1869. EThyella acrocephala (Meisn.) House, Bull. Torrey Bot. Club 33:313. 1906. Tipo: BRASIL. São PAULO: Rio Pardo, "in campis arenosis", X-1826, L. Riedel 574 (holótipo foto LE!; isótipo NY!).

Figuras. $6 \mathrm{j}-1$

Espécie caracterizada principalmente pelo hábito subarbustivo, inflorescências subsésseis glomeruliformes e multifloras. Pode ser confundida com J. fusca (Meisn.) Hallier f. (conhecida somente no Distrito Federal, Goiás, Bahia e Minas Gerais), mas esta última se diferencia pela inflorescência séssil ( $v s$. pedúnculo 2-4 mm compr.), com até 10 flores (vs. ca. de 20 flores).

Jacquemontia sphaerocephala é encontrada na Bolívia (Wood et al. 2014) e Brasil, ocorrendo em vegetação de Cerrado dos Estados de Mato Grosso, Mato Grosso do Sul, Goiás, Distrito Federal, Bahia, Minas Gerais e São Paulo (Flora do Brasil 2020, em constr.). Registrada em São Paulo apenas pelo material-tipo de J. acrocephala, coletado em 1826 e referido para Rio Pardo. Presume-se que o município da coleta seja São José do Rio Pardo, Santa Bárbara do Rio Pardo ou Santa Cruz do Rio Pardo. Áreas rurais de São José do Rio Pardo foram visitadas, mas nenhuma espécie de Jacquemontia foi encontrada, atualmente a 
agropecuária é predominante no local. Coletada com flores em outubro.

De acordo com a lista vermelha de espécies vegetais ameaçadas do Estado de São Paulo (SMA 2016), Jacquemontia sphaerocephala está presumivelmente extinta no Estado, pois enquadra-se no critério 1 , referente a ausência de novos registros nos últimos cinquenta anos.

Material examinado selecionado: BRASIL. SÃo Paulo: Rio Pardo, X-1826, L. Riedel 574 (NY!).

Material examinado adicional: BRASIL. BAHIA: Rio Corrente, Barreiras, 15-IV-1966, H.S. Irwin et al. 14888, (NY, SP, SPF, UB). Distrito Federal: Brasília, Samambaia, Parque Boca da Mata, 10-XII-1995, C. Aoki 260 (CEN, SP). GoiÁs: Chapadão do Céu, 28-I-2009, C. Aoki 345 (CGMS, SP); Serra Geral. 7-IV-1963, H.S. Irwin et al. 14520 (NY, SP). MinaS Gerais: Delfinópolis, 24-III-2000, A.C.B. Silva 380 (SP, SPSR); Unaí, Formoso, Km 122 da estrada, 14-III-1987, M. Brandão 12232 (PAMG, SP).

11. Jacquemontia sphaerostigma (Cav.) Rusby, Bull. of Torrey Bot. Club 26: 151. 1899. 三 Convolvulus sphaerostigma Cav., Icon. etDescriptiones Plantarum 5: 54. pl. 481. 1799. 三Jacquemontia hirsuta Choisy, Mém. Soc. Phys. Genève 8: 141. 1838, nom. illeg. Tipo: MÉXICO. «Habitat in Mindanao, floret Decembri; et in diversorio vulgo del Alto Camaron Regni mexicani ubi floret April et Maio. Vidi sccum in eodem herbario." (holótipo foto MA!; fragmento foto F!). Convolvulus apocynoides Schltdl. \& Cham., Linnaea 5: 117. 1830. 三Jacquemontia apocynoides (Schltdl. \& Cham.)Urb., SymbolaeAntillanae 8: 560. 1921. Tipo: MÉXICO. Vera CRUZ: X-1828, C.J.W. Schiede \& F. Deppe s.n. (holótipo foto HAL!). Convolvulus coeruleus Martens \& Galeotti, Bull. Acad. Brux. 12:254. 1845. Tipo: MÉXICO. OAXACA: Cordillera, 1840, H.G. Galeotti 1359 (lectótipo foto BR[0000006991944]!, designado por McDonald [1993]; isolectótipos foto BR[0000006992279], foto G!; K!; P!). Jacquemontia evolvuloides Moric. var. brevipedunculata Meisn., in Mart., F1. Bras. 7: 307. 1869. Tipo: BRASIL. Minas GeraIs: Lagoa Santa, 25-IV-1865, E. Warming s.n. (holótipo foto BR!, isolectótipo P!). Jacquemontia hirsuta var. trichodonta Meisn., in Mart., Fl. Bras. 7: 299. 1869. Tipo: BRASIL. Minas Gerais: 1840, P. Claussen 71 (síntipo foto BR!); Barbacena, 24-VI-1863, E. Warming s.n. (síntipo foto BR!). Jacquemontia hirsuta var. pohlii Meisn., in Mart., F1. Bras. 7: 299. 1869. Tipo: BRASIL. 1939, J.B.E. Pohl (holótipo foto BR!; isótipos foto F!, foto M!). Jacquemontia hirsuta var. adenotricha Hassl., Repert. Spec. Nov. Regni Veg. 9: 193. 1911. Tipo: PARAGUAI. "pr. Flumen Jejui Guazú”, XII-1898, E. Hassler 5694 (síntipos G; K!); Concepción, Villa-Sana “zwischen Rio Apa und Rio Aquidaban", 21-I-1909, K. Fiebrig 4663 (síntipos G; K!). Jacquemontia viscidulosa Hoehne, Anexos Mem. Inst. Butantan, Secc. Bot. 1: 51. pl. 7. 1922. Tipo: BRASIL. Mato Grosso: Porto Esperança, IX1914, J.G. Kuhlmann 1272 (lectótipo R!, designado por Buril \& Staples [2017]; isolectótipos SP!, LIL). Jacquemontia agricola Rusby, Mem. New York Bot. Gard. 7: 337. 1927. Tipo: BOLÍVIA. Canamina, VII1922, H.H. Rusby 80 (holótipo NY!). Jacquemontia laxiflora O'Donell, Lilloa 30: 14 (1960). Tipo: ARGENTINA. Missiones: Dep. San Ignacio, Puerto Nuevo, 12-III-1946, G.J. Schwartz 2211 (holótipo LIL!; isótipo foto S!).

Figuras 5 n-o, 11

Espécie caracterizada principalmente pela presença de tricomas forcados, estrelados 3-radiados e glandulares, inflorescência umbeliforme a corimbiforme, sépalas do mesmo tamanho, lanceoladas e com ápice acuminado. A densidade de tricomas glandulares nesta espécie é variável, estão presentes nos pedúnculos, bractéolas, pedicelos e sépalas, ou até mesmo nos ramos e margens das folhas em alguns indivíduos. Poucos espécimes examinados não possuem tricomas glandulares, como observado em A.C. Brade 5568 e 6986 (SP, SPF) e A. Usteri $10 b$ (SP, SPF). O'Donell (1960a) também notou a ausência de tricomas glandulares em poucos indivíduos e considerou como uma anomalia da espécie.

Jacquemontia evolvuloides (Moric.) Meisn. é a espécie mais semelhante à $J$. sphaerostigma, distinguindo-se pela inflorescência em monocásios, composta por uma a três flores, possui ampla distribuição na América Tropical, no Brasil ocorre na Caatinga e Cerrado até o Estado de Minas Gerais.

Jacquemontia sphaerostigma apresenta ampla distribuição na América tropical, desde o México até a América do Sul, nos países da Colômbia, Peru, Bolívia, Venezuela (Robertson 1971) e no Brasil é registrada em todas as Regiões, exceto a Região Sul (Flora do Brasil 2020, em constr.). Em São Paulo ocorre em vegetação de Cerrado e Floresta Estacional Semidecídua, geralmente associada a afloramentos rochosos. Coletada com flores e frutos de fevereiro a novembro. 
De acordo com a metodologia de Mamede et al. (2007) a espécie foi classificada como menos preocupante (LC) no Estado São Paulo, pois não enquadra-se em nenhum dos critérios.

Material examinado selecionado: BRASIL. São PAULO: Araraquara, 8-V-2004, J.L.M. Aranha Filho \& B.L. Tacarambi 9 (UEC); Atibaia, Nativo na saída da cidade, rumo à Piracaia, 25-VII-1992, G. Hashimoto 20504 (SP, GHSP); Botucatu, margem da Rodovia Municipal (Estrada Roberto), 18-IV-1995, R. SimãoBianchini et al. 677 (SP); Cabreúva, entre a Rodovia e o Rio Tietê, 4-V-1995, W. Marcondes-Ferreira et al. 1130 (SP, UEC); Campinas, subdistrito de Barão Geraldo, UNICAMP (campo de milho), 7-IX-1957, A.S. Grotta 211 (SPF); Castilho, próximo da foz do Rio Aguapei., s.d., F.C. Hoehne s.n. (SP 20543); Cunha, Serra da Cunha, 6-II-1965, G. Eiten et al. 5835 (SP, SPF); Igaçaba, Morro Solteiro, 17-IV-2003, D. Sasaki et al. 319 (SP, SPF); Jundiaí, 21-IV-1995, M. Batalha et al. 380 (SP); Mairiporã, 19-XI-1962, P.M. Camargo \& G. Marini 85 (SP, SPF, SJRP); Mogi Guaçu, margem do Rio Mogi Guaçu, 24-VI-1965, G. Marinis 338 (SP, SJRP); Mogi Mirim, 27-III-1918, F.C. Hoehne s.n. (SP 2571, SPF); Onda Verde, Fazenda São João, 1-II-1907, A. Usteri 10 (SP, SPF); Paraguaçu Paulista, $6 \mathrm{~km}$ orth of city Paraguaçu Paulista, 24-III-1912, A.C. Brade 5568 (SP, SPF); Pedregulho, Usina de Estreito, encosta à margem da represa, próxima à barragem, X-1987, R.S. Bianchini 24 (SPF); Pirassununga, Cerrado das Emas, $22^{\circ} 02^{\prime} \mathrm{S}$, 47³0'W, 6-IV-2000, D. Sasaki 46 (SPF); São José do Rio Preto, 8-VIII-1995, M.R. Pereira-Noronha et al. 1629 (SP); São Paulo, Butantan, 22-III-2004, D. Sasaki \& M.F.A. Calió 999 (SPF); Suzanápolis, na divisa com Pereira Barreto, junto ao rio São José dos Dourados, 11-IV-1996, N.T. Ranga \& A.A. Rezende 371 (SJRP); Valinhos, Rodovia Valinhos-Itatiba, 17-IV-2003, D. Sasaki et al. 319 (SPF).

Ilustrações: como J. hirsuta em Meisner (1869, tab. 110); Robertson (1971, p. 237, fig. 12 E-F); SimãoBianchini \& Pirani (1997, p. 143, figs. 58-60).

12. Jacquemontia spiciflora (Choisy) Hallier f., Bot. Jahrb. Syst. 16: 543. 1893. 三Ipomoea spiciflora Choisy, Mém. Soc. Phys. Genève 8: 132. 1838. Tipo: BRASIL. Pará, A. Ferreira s.n. (holótipo $\mathrm{P}[\mathrm{P} 00723265]$ !; isótipo $\mathrm{P}[03537536] !)$.

Figuras 8 i-n, 9, 10 d-f

Entre as espécies do gênero encontradas no Estado de São Paulo, apenas Jacquemontia spiciflora, J. sphaerocephala e J. selloi possuem hábito subarbustivo, espécies características do Cerrado. Jacquemontia spiciflora se distingui pela inflorescência espiciforme e sépalas glabras com ápice arredondado a obtuso, enquanto $J$. sphaerocephala possui dicásios glomeruliformes, sépalas tomentosas com ápice acuminado e $J$. selloi apresenta dicásios corimbiformes e sépalas velutinas a pubescentes com ápice também acuminado.

Conhecida como endêmica do Brasil, ocorre nos Estados do Pará, Mato Grosso, Goiás, Minas Gerais e São Paulo (Flora do Brasil 2020, em constr.) em Cerrado de campo limpo e campo sujo, frequentemente associada a afloramento rochoso. Coletada com flores e frutos de dezembro a março.

Recomenda-se medidas de conservação para esta espécie, pois, apesar de não ser classificada como ameaçada no Estado São Paulo pela metodologia de Mamede et al. (2007), há poucos registros recentes na área de estudos e sua distribuição é restrita a em áreas de Cerrado.

Material examinado selecionado: BRASIL. São PAULO: Araraquara, Cerrado a margem Km 200, Estrada SP Araraquara, 1961, A. Lima 3703 (RB); Campinas, 10-II-1944, A. Macedo 258 (SP); Franca, 9-I-1993, $A$. Loefgren G. \& Edwall 2080 (SP); Jeriquara, Fazenda da Estiva, 17-III-1964, J. Mattos \& H. Bicalho 11577 (SP); Mogi Guaçu, Reserva Biólogica e Estação Experimental de Mogi Guaçu, Distrito de Martinho Prado, 17-III-1981, M. Oliveira \& W. Mantovani 31 (SP); Patrocínio Paulista, Patrocínio do Sapucaí, 14-I1893, A. Loefgren G. Edwall 2131 (SP); Pedregulho, Parque Estadual das Furnas do Bom Jesus, próximo à trilha do Chalé, 19-2-2013, M. Pastore et al. 237 (SP, PMSP, SPSF, UEC).

13. Jacquemontia tamnifolia (L.) Griseb., Fl. Brit. W. Ind. 474. 1862. 三Ipomoea tamnifolia L. Sp. P1. 1: 162. 1753. $\equiv$ Convolvulus tamnifolius (L.) G. Mey., Prim. Fl. Esseq. 95. 1818. $\equiv$ Thyella tamnifolia (L.) Raf., Fl. Tellur. 4: 84. 1838. Tipo: Ilustração em Dillenius, Hortus Elthamensis, p. 428, t. 318 f. 414. 1732 (lectótipo designado por Robertson [1971]). Convolvulus capitatus Desr., Encycl. 3: 554. 1789[1792]. 三Jacquemontia capitata (Desr.)G.Don, Gen. Hist. 4: 283. 1838. इIpomoea capitata (Desr.) Choisy, in DC. Prodr. Syst Nat. 9: 365. 1845. Tipo: SENEGAL. Goeffrory s.n. (holótipo P!). Convolulus ciliatus Vahl, Eclog. Amer. 2: 13. 1798. "Cajenna", J.P.B. von Rohr 109 (holótipo foto C[10009713]!; 

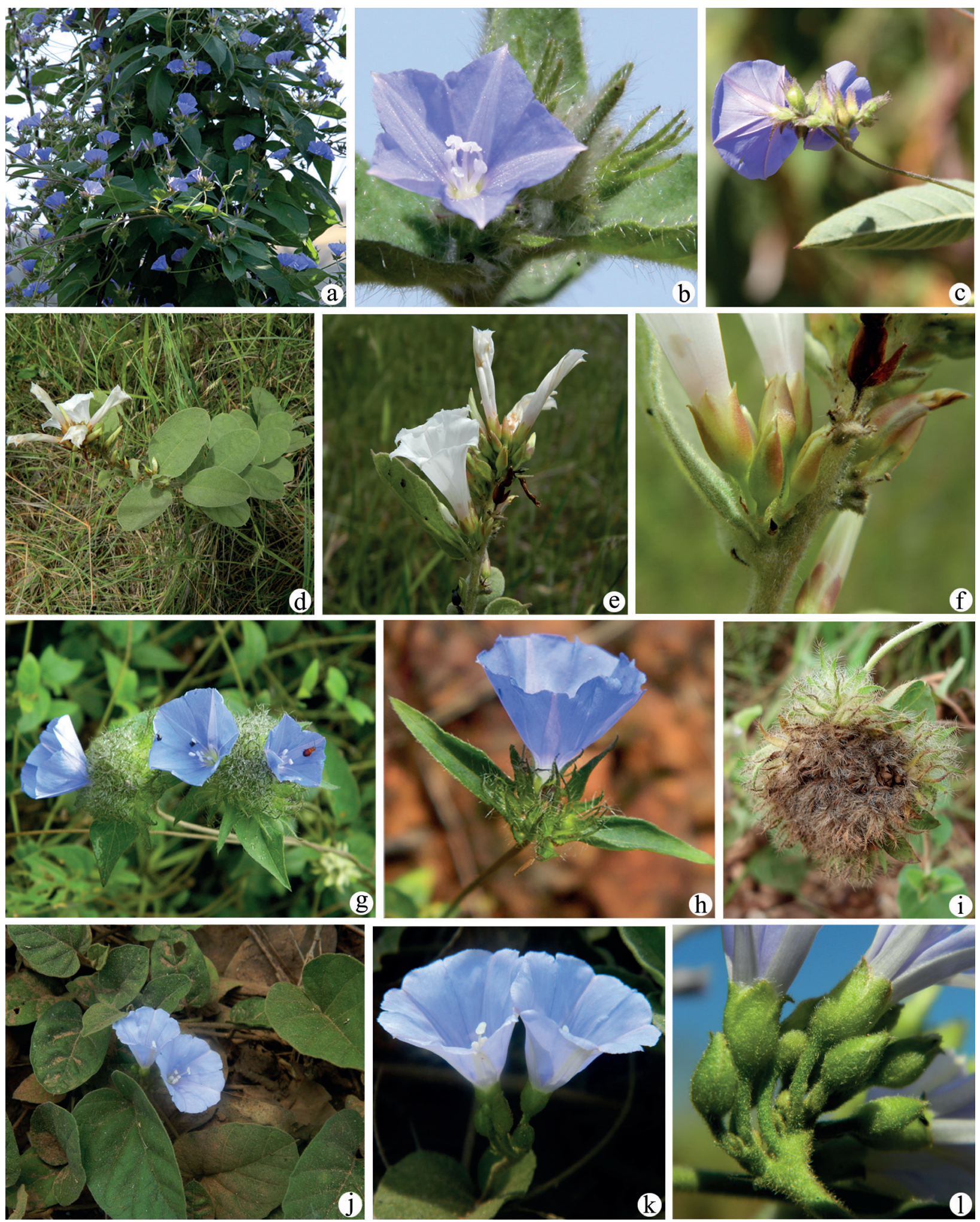

Figura 10. a-c. Jacquemontia sphaerostigma. a. Hábito. b. Flor em vista frontal. c. Inflorescência. d-f. Jacquemontia spiciflora. d. Hábito. e. Inflorescência. f. Detalhe das sépalas. g-i. Jacquemontia tamnifolia. g. Inflorescências. h. Flor em vista longitudinal. i. Cápsulas. j-1. Jacquemontia velutina. j. Hábito. k. Inflorescência. 1. Detalhe dos cálices. Fotos a-c H. Moreira.

Figure 10. a-c. Jacquemontia sphaerostigma. a. Habit. b. Flower in front view. c. Inflorescence. d-f. Jacquemontia spiciflora. d. habit. e. Inflorescence. f. Detail of the sepals. g-i. Jacquemontia tamnifolia. g. Inflorescences. h. Flower in longitudinal view. i. Capsules. j-1. Jacquemontia velutina. j. Habit. k. Inflorescence. 1. Detail of the calyxes. Photos a-c H. Moreira. 


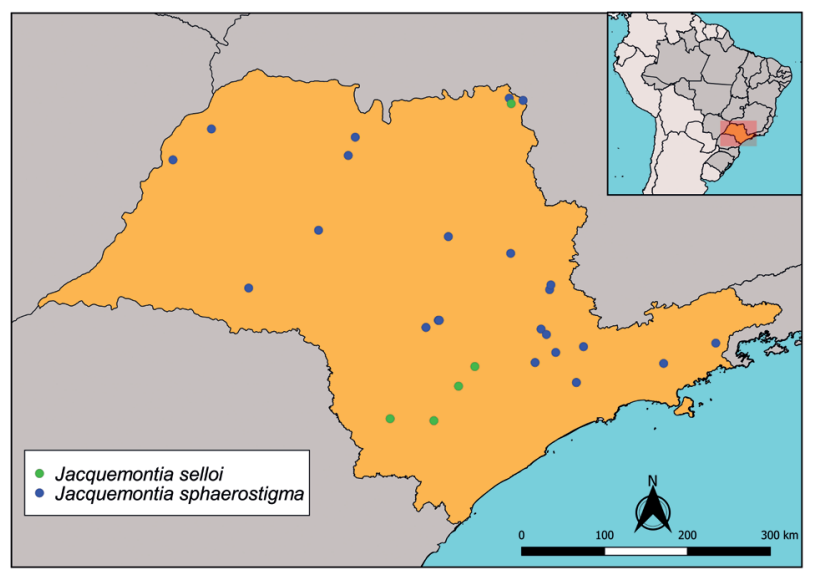

Figura 11. Mapa com a distribuição de Jacquemontia selloi e Jacquemontia sphaerostigma no Estado de São Paulo, Brasil.

Figure 11. Distribution map of Jacquemontia selloi and Jacquemontia sphaerostigma in São Paulo State, Brazil.

isótipo foto C[10009712]!). Convolvulus guineensis Schumach.,Bersk. Guin.Plant.p.90.1827. $\equiv I$ pomoea guineensis (Schumach.) G. Don, Gen. Hist. 4: 269. 1838. Tipo: GHANA. P. Thonning s.n. (lectótipo foto C[10003617], designado por Robertson [1971]; isolectótipos foto C[10003616]!, foto C[10003615]!). Convolvulus pycnanthus Hochst. ex Choisy, in DC. Prodr. Syst Nat. 9: 365. 1845.Tipos: SUDAN. Kordofan: Hogel, C.G.T. Kotschy 212 (holótipo G; isótipos foto BR!, foto HBG!, K!, foto M!, foto TUB!). Convolvulus praelongus S. Moore, Trans. Linn. Soc. London Bot. 4: 403. 1895. Tipo: BRAZIL. MATO Grosso, S. Moore 1101a (holótipo foto BM!). Jacquemontia macrocephala Brandegee, Zoe 5(10): 219. 1905. $\equiv$ Thyella macrocephala (Brandegee) House, Muhlenbergia 5: 68. 1909. Tipo: MÉXICO. Sinaloa: vicinity of Culiacan, 5-XI-1904, T.S. Brandegee s.n. (holótipo foto UC!; isótipos foto GH!, foto US!). Jacquemontia rondonii Hoehne, Anex. Mems. Inst. Butantan Sec. Bot. 1(6): 53. pl. 8. 1922. Tipo: BRASIL. Mato Grosso: Serra dos PacahasNovos, em região do Cautário Grande, III-1918, M.C. Rondon 2263 (holótipo R!; isótipo foto LIL!). Jacquemontiamattogrossensis Hoehne,Anex. Mems. Inst. Butantan Sec. Bot. 1(6): 54. pl. 9. 1922. Tipo: BRASIL. Mato Grosso: Porto Esperança, IX-1914, J.G. Kuhlmann 1273 (holótipo R!; isótipo foto LIL!).

Figuras 10 g-i, 4

Espécie reconhecida pelas cimeiras capituliformes, multifloras, bractéolas em três séries, sendo as duas externas foliáceas, sépalas lanceoladas, ciliadas e com ápice longo-acuminado. $\mathrm{O}$ indumento é hirsuto a glabrescente, formado por tricomas forcados ou simples, destacando-se entre a maioria das espécies do gênero que apresentam tricomas estrelados.

Semelhante morfologicamente à Jacquemontia bracteosa Meisn. (restrita ao nordeste brasileiro e Minas Gerais), porém esta diferencia-se pelo indumento tomentoso a velutino, formado por tricomas estrelados 3-radiados e forcados, bractéolas não foliáceas e inflorescência mais densa.

Entre as espécies do gênero, J. tamnifolia apresenta a maior distribuição geográfica, ocorrendo nas Américas desde o sudoeste dos Estados Unidos até a Argentina e se espande para o leste, oeste e sul da África, Madagascar e Ilhas Mascarenhas (Robertson 1971). No Brasil é encontrada em todas as regiões, em diversos tipos de vegetação, incluindo áreas alteradas (Flora do Brasil 2020, em constr.). Em São Paulo foi registrada apenas em uma área antropizada do Instituto de Botânica, crescendo espontaneamente. Coletada com flores em maio.

Apesar de haver apenas um registro desta espécie no Estado de São Paulo, sua avaliação estadual não é aplicável (IUCN 2012), pois é considerada uma espécie ruderal que possivelmente foi trazida de outras áreas através do transporte ocasional de suas sementes.

Material examinado selecionado: BRASIL. S̃̃o Paulo: São Paulo, V-2006, R. Simão-Bianchini et al. 1593 (SP).

Material examinado adicional: BRASIL. BAHIA: Bom Jesus da Lapa, ca. $14 \mathrm{~km}$ S do entroncamento para Bom Jesus da Lapa na estrada para Malhada, 11-II-2000, L.P. Queiroz 5856 (HUEFS). Espírito Santo: Conceição da Barra, 9-VI-2003, G. Hatschbach et al. 75073 (MBM). Mato Grosso: Barão de Melgaço, RPPN SESC Pantanal, 11-III-2002, S.R. Zaniolo et al. 200 (MBM). Mato Grosso do Sul: Corumbá, Córrego Zé Dias, $17^{\circ} 52^{\prime} 08.3^{\prime \prime S}, 5^{\circ} 31^{\prime} 26.7^{\prime \prime W}, 5-\mathrm{V}-2001$, G.A. Damasceno Jr. et al. 2329 (SP, COR, MBM). MinAS GERAIs: Inhapin, 1-II-1967, J. Lindeman H. haas 4576 (MBM). Pará: Canaã dos Carajás, Floresta Nacional de Carajás, Serra Sul, S11B, 6²1'21"S, 50²3'27"W, 4-V-2016, M. Pastore et al. 303 (MG). Rio GRANDE do Norte: São Miguel do Gostoso, Paraíso, 19-V-2007, G.B.C. Paterno \& M.I.B. Loiola 170 (SP, UFRN).

Ilustrações: O’Donell (1960, p. 25, fig 7, A-F); Verdcourt (1963, p. 36, fig. 10 1-7); Robertson (1971, p. 239, fig. 13 A-F); Deroin (2001, p. 85, fig. 25 1-11), Ferreira (2013, p. 295, fig. 3 E-F). 


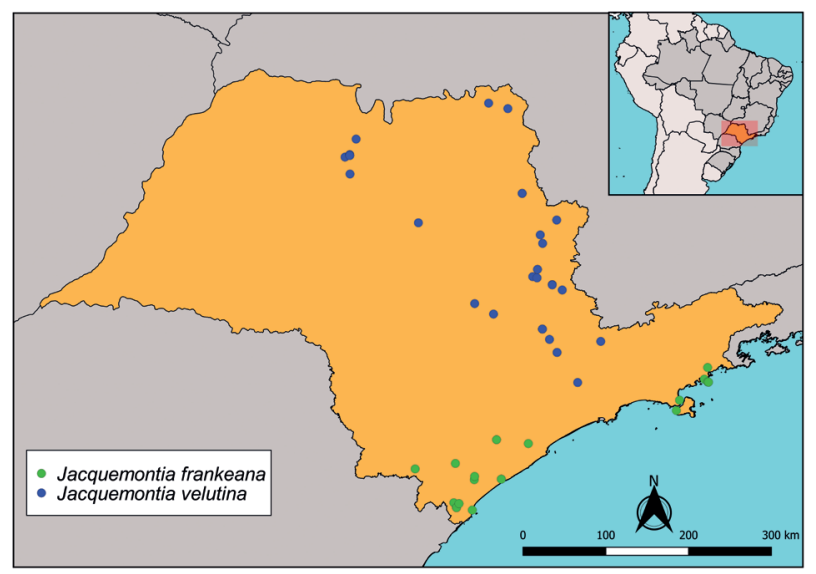

Figura 12. Mapa com a distribuição de Jacquemontia frankeana e Jacquemontia velutina no Estado de São Paulo, Brasil.

Figure 12. Distribution map of Jacquemontia frankeana and Jacquemontia velutina São Paulo State, Brazil.

14. Jacquemontia velutina Choisy, in DC. Prodr. Syst Nat. 9: 398. 1845. Tipo: BRASIL. 1839, Herb. Mart. n. 67, J. Pohl s.n. (lectótipo foto BR[0000005843947]!, aqui designado; isolectótipos foto BR[0000005843619]!, foto M!).

Figuras $10 \mathrm{j}-1,12$

O lectótipo BR0000005843947 foi designado para $J$. velutina, pois é o único espécime que contém Mart. 67 escrito na etiqueta, como referido no protólogo da espécie.

Espécie reconhecida pelos ramos e folhas velutinas, dicásios corimbiformes, sépalas ovadas a obovadas, todas iguais em tamanho, pubescentes, com ápice arredondado a obtuso e pelos grãos de pólen tricolpados. Entre as espécies da área de estudo, $J$. blanchetii é a mais semelhante morfologicamente à $J$. velutina, mas a primeira distingue-se pelos ramos e folhas glabrescentes, inflorescência umbeliforme, sépalas glabras e grãos de pólen pantocolpados.

Jacquemontia velutina Griseb. (non Choisy) foi publicada em 1979, portanto um nome ilegítimo. Trata-se de um sinônimo de J. lorentzii (Kuntze) Peter ex O'Donell (Argentina, Bolívia e Paraguai), reconhecido por O'Donell (1960b) e muito diferente morfologicamente de $J$. velutina Choisy.

Jacquemontia velutina é registrada na Bolívia em Santa Cruz (Wood et al. 2014) e Brasil nos Estados do Pará, Tocantins, Goiás, Distrito Federal, Mato Grosso do Sul, Minas Gerais, Bahia, São Paulo e Rio de Janeiro (Flora do Brasil 2020, em constr.). Em São Paulo é encontrada em vegetação de Cerrado e Floresta Estacional Semidecídua, em áreas de campo, bordas de mata e beira de estradas. Coletada com flores e frutos de março a agosto.

Classificada como menos preocupante (LC) no Estado São Paulo, de acordo com a metodologia proposta por Mamede et al. (2007), pois não enquadra-se em nenhum dos critérios.

Material examinado selecionado: BRASIL. São PAULO: Campinas, Avenida Brasil, 15-V-1937, J. Sandoro s.n. (IAC987); Águas de São Pedro, 11-IV-1993, S. Bianchini \& R. Simão-Bianchini 14 (SP); Buritizal, Fazenda Buritis, 5-V-1995, W. Marcondes-Ferreira et al. 1185 (SP, PMSP); Cajuru, Fazenda Rio Grande, 25-IV-2000, M.C. Gaglionone \& G.A.R. Melo s.n. (SP 347600); Campinas, Mata do Laboratório Nacinoal de Luz Sincrotron, 8-IV-2004, S.R., Morbiolo \& R.L. Fonseca 193 (ESA); Casa Branca, beira da Rodovia SP-340, km 217, 24-IV-2013, M. Pastore 280 (SP, HRCB); Jundiaí, 12-IV-1994, L.C. Bernacci et al. 36 (SP, IAC); Matão, Fazenda Cambuhy, 12-IV-1994, K.D. Barreto et al. 2274 (SP, ESA); Mogi Guaçu, Reserva Biológica e Extação Experimental de Mogi Guaçu, beira da Mata da Mariana, 23-IV-2013, M. Pastore 278 (SP); Onda Verde, beira da Estrada Municipal Gadbyn Mefle, 9-V-2013, M. Pastore 286 (SP, BOTU, SJRP); Pedregulho, Parque Estadual das Furnas do Bom Jesus, 18-III-1994, W. MarcondesFerreira et al. 799 (SP); Piracaia, Rodoanel, trecho Sul, plantio compensatório, 18-IV-2012, R.T. Shirasuna \& M. Medeiros 3023 (SP); Piracicaba, Bairro Jardim Europa, 14-IV-1966, W.R. Accorsi s.n. (ESA4934); Potirendaba, 24-IV-1999, L.R. Gimenez \& D. Almella 11 (SJRP); São José do Rio Pardo, 2-IV-1943, F. Glasauer 601 (SPSF); São José do Rio Preto, Estação Ecológica de São José do Rio Preto e Mirassol, 10-V-2013, M. Pastore 288 (SP, PMSP, UEC); São Paulo, Alto da Lapa, 6-IV-1942, W. Hoehne 1042 (SP, SPF); Vinhedo, Rodovia Ahanguera, $\mathrm{km} 74$, 5-IV-2003, J.R. Guillaumon 33916 (SPSF).

Ilustrações: Meisner (1869, t. 106-I).

\section{Agradecimentos}

À Coordenação de Aperfeiçoamento de Pessoal de Nível Superior (CAPES) pela bolsa concedida à primeira autora. Ao Conselho Nacional de Desenvolvimento Científico e Tecnológico (CNPq) e Fundação de Amparo à Pesquisa do Estado de São Paulo (FAPESP) pelo financimento do trabalho nos herbário K e P realizado pela segunda autora através do Projeto REFLORA (CNPq 563570/2010-5; FAPESP 
2010/52490-3). Aos curadores dos herbários BOTU, ESA, HB, HRCB, IAC, K, MBM, P, PMSP, R, RB, SJRP, SP, SPF, SPSF e UEC pelo acesso às coleções. Ao Vitor Gedha Pescara pelas ilustrações cobertas a nanquim. Ao Rafael Melo de Brito pela elaboração dos mapas. À Fernanda Satori Petrongari pelas contribuições. À Andréa Onofre Araújo, à Priscila Porto Alegre Ferreira e aos revisores anônimos pelas contribuições e revisão do trabalho.

\section{Literatura citada}

APG (Angiosperm Phylogeny Group). 2016. An update of the Angiosperm Phylogeny Group classification for the orders and families of flowering plants: APG IV. Botanical Journal of the Linnean Society 181: 1-20.

Athiê-Souza, S.M., Staples, G., Zickel, C.S. \& Buril, MT. 2017. Towards a Better Understanding of the Tribe Aniseieae: Revisiting Aniseia and Iseia (Convolvulaceae). Systematic Botany 42(3): 590-605.

Austin, D.F. 1973. The American Erycibeae (Convolvulaceae): Maripa, Dicranostyles and Lysiostyles. Systematics. Annals of the Missouri Botanical Garden 60: 306-412.

Austin, D.F. 1982. Convolvulaceae. In: G. Harling \& B. Sparre (eds.). Flora of Ecuador. University of Götenborg, Riksmuseum, Göteborg, Stockholm, v. 15, pp. 1-98.

Austin, D.F. 1998. Parallel and convergent evolution in the Convolvulaceae. In: P. Mathews \& M. Sivadasan (eds.). Biodiversity and taxonomy of tropical flowering plants. Mentor Books, Calicut, pp. 201-234.

Austin, D. F. 1999. The genus Aniseia (Convolvulaceae). Systematic botany 23: 411-420.

Austin, D.F. \& Cavalcante, P.B. 1982. Convolvulaceas da Amazônia. Boletim do Museu Paraense Emilio Goeldi 36: 1-134.

Buril, M.T. 2013. Sistemática e Filogenia de Jacquemontia Choisy (Convolvulaceae). Tese de Doutorado, Universidade Federal de Pernambuco, Recife.

Buril, M.T., Simões, A.R., Carine, M. \& Alves, M. 2015. Daustinia, a replacement name for Austinia (Convolvulaceae). Phytotaxa 197: 60.

Buril, M.T. \& Staples, G. 2017. Lectotypification of names for Jacquemontia (Convolvulaceae) species. Nordic Journal of Botany. Accepted Author Manuscript.

Cheek, M., \& Simão-Bianchini, R. 2013. Keraunea gen. nov. (Convolvulaceae) from Brazil. Nordic Journal of Botany 31: 453-457.

Choisy, J.D. 1834. Convolvulaceae orientales. Mémoires de la Société de Physique de Genève 6: 385-502.

Choisy, J.D. 1837. Convolvulaceae Mémoires de la Société de Physique de Geneve 8: 122-164.
Choisy, J.D. 1845. Convolvulaceae. In: A. Candolle (ed.). Prodromus systematics naturalis regni vegetabilis. Sumptibus Sociorum Treuttel et Würtz, Parisii, v. 9, pp. 323-465.

Deroin, P.T. 2001. Convolvulaceae. In: P.H. Morat. (ed.). Flore de Madagascar et des comores. Imprimerie officielle, Paris, v. 171, pp. 11-287.

Falcão, J.I.A. 1971. As Convolvulaceas do Estado de São Paulo. Loefgrenia 52: 1-20.

Ferreira, P.P.A. 2013. Convolvulaceae na Região Sul do Brasil. Tese de Doutorado, Universidade Federal de Rio Grande do Sul, Rio Grande do Sul.

Flora do Brasil 2020 (em construção). 2017. Jardim Botânico do Rio de Janeiro. Disponível em http:// floradobrasil.jbrj.gov.br/ (18-09-2017).

Hallier, H.J.G. 1893. Versuch einer naturlichen gliederung der Convolvulaceen auf morphologischer und anatomischer Grundlage. Botanische Jahrbücher für Systematik, Pflanzengeshichte und Pflanzengeographie 16: 453-591.

Hallier, H.J.G. 1899. Zur Convolvulaceenflora Amerika's. Jahrbuch der Hamburgischen Wissenschaftlichen Anstalten 16: 19-56.

Hoehne, F.C. 1922. Convolvuláceas dos Herbários: Horto "Oswaldo Cruz", Museu Paulista e Comissão Rondon. Memórias do Instituto de Butantan Secção de Botânica 6: 5-83.

IUCN (International Union for Conservation of Nature). 2001. Red List Categories and Criteria: Version 3.1. IUCN, Gland, Switzerland.

IUCN (International Union for Conservation of Nature). 2012. Guidelines for application of IUCN Red List Criteria at Regional Levels: Version 3.1. Second edition. IUCN Species Survival Commission. IUCN, Gland, Switzerland.

Judd, W.S., Campbell, C.S., Kellog, E.A., Stevens, P.F. \& Donoghue, M.J. 2009. Plant Systematics: A phylogenetic approach. 3 ed. Sunderland, Sinauer Associates.

Krapovickas, A. 2009. Novedades en Convolvuláceas argentinas. Bonplandia 18: 57-64.

Mamede, M.C., Souza, V.C., Prado, J., Barros, F., Wanderley, M.G.L \& Rango, J.G. (orgs.). 2007. Livro Vermelho das espécies Vegetais Ameaçados do Estado de São Paulo. Instituto de Botânica, São Paulo.

Meisner, C.F. 1869. Convolvulaceae. In: C.P.F. Martius \& A.G. Eichler (eds.). Flora Brasiliensis. F. Flischer, Lipsiae, v. 7, pp. 199-370.

Moricand, S. 1838. Plantes Nouvelles d'Amérique v. 27. Imprimerie de Jules- Gme Fick, Genève.

O'Donell, C.A. 1953. Convolvulaceae americanas nuevas o criticas IV. Lilloa 26: 353-400.

O'Donell, C.A. 1960a. La especies de Jacquemontia de Peru. Lilloa 20: 71-106. 
O'Donell, C.A. 1960b. Convolvulaceas argentinas II. Lilloa 30: 5-39.

Olmstead, R. G., Bohs, L., Migid, H. A., SantiagoValentin, E., Garcia, V. F., \& Collier, S. M. 2008. A molecular phylogeny of the Solanaceae. Taxon 57: 1159-1181.

Pastore, M. \& Simão-Bianchini, R. 2015. Taxonomic novelties in Jacquemontia Choisy (Convolvulaceae) from Southeastern Brazil. Phytotaxa 221: 193-197.

Piedade-Kill, L.H. \& Ranga, N.T. 2000. Biologia floral e sistema de reprodução de Jacquemontia multiflora (Choisy) Hallier f. (Convolvulaceae). Revista Brasileira de Botânica 23: 37-43.

QGIS, D. T. 2016. "Quantum GIS geographic information system." Open Source Geospatial Foundation Project 45.

Rhui-Cheng, F. \& Staples, G. 1995. Convolvulaceae In: Flora of China Editorial Committee. Flora of China. Missouri Botanical Garden Press, St. Louis, v. 16, pp. 271-325.

Robertson, K.R. 1971. Arevision of the genus Jacquemontia (Convolvulaceae) in North and Central America and the West Indies. Ph. D. Dissertation Washington University, St Louis.

Simão-Bianchini, R. 2009. Flora de Grão-Mogol, Minas Gerais: Convolvulaceae. Boletim de Botânica Universidade de São Paulo 27: 33-41.

Simão-Bianchini, R. \& Pirani, J.R. 1997. Flora da Serra do Cipó, Minas Gerais: Convolvulaceae. Boletim de Botânica Universidade de São Paulo 16: 125-149.

Simão-Bianchini, R. \& Pirani, J.R. 2005. Duas novas espécies de Convolvulaceae de Minas Gerais, Brasil. Hoehnea 32: 295-300.

Simão-Bianchini, R. \& Silva, C.V. 2011. Convolvulaceae. In: M.G.L. Wanderley, G.J. Shepherd, S.E Martins, T.E.M.D. Estrada, R.P. Romanini, I. Koch, J.R. Pirani, T.S. Melhem, A.M.G. Harley, L.S. Kinoshita., M.A.G. Magenta, H.M.L. Wagner, F. Barros, L.G. Lohmann, M.C.E. Amaral, I. Cordeiro, S. Aragaki, R.Simão-Bianchini, \& G.L. Esteves (orgs.). Checklist of Spermatophyta of the São Paulo State, Brazil. Biota Neotropica v. 11 n. 1, pp. 193-390.

Simões, A.R. \& Staples, R. 2017. Dissolution of Convolvulaceae tribe Merremieae and a new classification of the constituent genera. Botanical Journal of the Linnean Society 183: 561-586.
Secretaria do Meio Ambiente do Estado de São Paulo. 2016. Resolução SMA n. 57, de 5-VI-2016. Lista de espécies da flora ameaçadas de extinção no Estado de São Paulo. Diário Oficial do Estado de São Paulo, Poder Executivo, São Paulo, 05-VI-2016. Seção I, 126(103), pp. 55-57.

Staples, G.W., Carine, M. \& Austin, D.F. 2008. Convolvulaceae Pollen Atlas. Disponível em http://cals.arizona.edu/ herbarium/sites/cals.arizona.edu.herbarium/files/old site/assoc/projects/convolv/Convolvulaceae_Pollen Atlas.htm (acesso em 12-IV-2017).

Staples, G. 2012. Convolvulaceae - the morning glories and bindweeds. Disponível em http://convolvulaceae. myspecies.info/node/9 (acesso em 26-I-2017).

Stefanović, S., Krueger, L. \& Olmstead, R.G. 2002. Monophyly of the Convolvulaceae and circumscription of their major lineages based on DNA sequences of multiple choroplast loci. American Journal of Botany 89: 1510-1522.

Stefanović, S., Austin, D.F., \& Olmstead, R.G. 2003. Classification of Convolvulaceae: A phylogenetic Approach. Systematic Botany 28: 797-806.

Thiers B. (continuamente atualizada). 2017. Index herbariorium: A global directory of publish herbaria and associated staff. New York Botanical Garden's Virtual Herbarium. Disponível em http://sciweb.nybg.org/ science2/IndexHerbariorum.asp (acesso em 14-V-2017).

Verdcourt, B. 1963. Convolvulaceae. In: C.E. Hubbard \& E. Milne-Redhead (eds.). Flora of tropical east Africa. Crown Agents for Overseas Governments \& Administrations, London, pp. 1-161.

Wanderley, M.G.L., Shepherd, G.J., Melhem T.S. \& Giulietti, A.M. (coords.). 2005. Flora Fanerogâmica do Estado de São Paulo. São Paulo, Rima \nstituto de Botânica de São Paulo, v. 4.

Wood, J.R.I., Simão-Bianchini, R. \& Fuentes-Claros, A. 2014. Convolvulaceae. In: B.M. Jørgensen, M. Nee \& S.G. Beck (eds.). Catálogo de las Plantas Vasculares de Bolivia. Missouri Botanical Garden Press, St Louis, pp. 520-531.

Wurdack, J.J. 1970. Erroneous data in Glaziou Collections of Melastomataceae. Taxon 19: 911-913.

Yang, S. \& Buril, M.T. 2012. A new alien distribution record and addition to the flora of Taiwan: Jacquemontia polyantha. Taiwania 57: 418-421. 